\title{
Analysis of microplastics in drinking water and other clean water samples with micro-Raman and micro-infrared spectroscopy: minimum requirements and best practice guidelines
}

\author{
Darena Schymanski ${ }^{1,2} \cdot$ Barbara E. Oßmann ${ }^{3} \cdot$ Nizar Benismail $^{4} \cdot$ Kada Boukerma $^{5} \cdot$ Gerald Dallmann $^{6} \cdot$ Elisabeth von \\ der Esch ${ }^{7}$. Dieter Fischer ${ }^{8}$. Franziska Fischer ${ }^{8}$ - Douglas Gilliland ${ }^{9} \cdot$ Karl Glas $^{10} \cdot$ Thomas Hofmann $^{10}$. \\ Andrea Käppler ${ }^{6}$. Sílvia Lacorte ${ }^{11}$ • Julie Marco ${ }^{12} \cdot$ Maria EL Rakwe $^{5}$. Jana Weisser ${ }^{10}$ Cordula Witzig $^{13}$. \\ Nicole Zumbülte ${ }^{13}$. Natalia P. Ivleva ${ }^{7}$ (iD
}

Received: 9 April 2021 / Revised: 17 June 2021 / Accepted: 22 June 2021 / Published online: 20 July 2021

(C) The Author(s) 2021

\begin{abstract}
Microplastics are a widespread contaminant found not only in various natural habitats but also in drinking waters. With spectroscopic methods, the polymer type, number, size, and size distribution as well as the shape of microplastic particles in waters can be determined, which is of great relevance to toxicological studies. Methods used in studies so far show a huge diversity regarding experimental setups and often a lack of certain quality assurance aspects. To overcome these problems, this critical review and consensus paper of 12 European analytical laboratories and institutions, dealing with microplastic particle identification and quantification with spectroscopic methods, gives guidance toward harmonized microplastic particle analysis in clean waters. The aims of this paper are to (i) improve the reliability of microplastic analysis, (ii) facilitate and improve the planning of sample preparation and microplastic detection, and (iii) provide a better understanding regarding the evaluation of already existing studies. With these aims, we hope to make an important step toward harmonization of microplastic particle analysis in clean water samples and, thus, allow the comparability of results obtained in different studies by using similar or harmonized methods. Clean water samples, for the purpose of this paper, are considered to comprise all water samples with low matrix content, in particular drinking, tap, and bottled water, but also other water types such as clean freshwater.
\end{abstract}

Keywords Microplastic $\cdot$ Micro-Raman spectroscopy $\cdot$ Micro-(FT)IR spectroscopy $\cdot$ Bottled water $\cdot$ Drinking water $\cdot$ Clean water

Darena Schymanski and Barbara E. Oßmann contributed equally to this work.

Natalia P. Ivleva

natalia.ivleva@tum.de

1 Chemical and Veterinary Analytical Institute Münsterland-Emscher-Lippe (CVUA-MEL), Joseph-König-Straße 40, 48147 Münster, Germany

2 Institute of Food Chemistry, Westfälische Wilhelms-Universität Münster, Corrensstr. 45, 48149 Münster, Germany

3 Bavarian Health and Food Safety Authority, Eggenreuther Weg 43, 91058 Erlangen, Germany

4 Nestle Quality Assurance Center Vittel, 1020 Avenue Georges Clemenceau, 88800 Vittel, France

5 Ifremer, REM/RDT/LDCM, 29280 Plouzané, France

6 SGS Institut Fresenius GmbH, Königsbrücker Landstr. 161, 01109 Dresden, Germany
7 Institute of Hydrochemistry, Chair of Analytical Chemistry and Water Chemistry, Department of Chemistry, Technical University of Munich, Elisabeth-Winterhalter-Weg 6, 81377 Munich, Germany

8 Leibniz Institute of Polymer Research Dresden (IPF), Hohe Straße 6, 01069 Dresden, Germany

9 Joint Research Centre (JRC), European Commission, 21027 Ispra, Italy

10 Chair of Food Chemistry and Molecular Sensory Science, Technical University of Munich, Lise-Meitner-Straße 34, 85354 Freising, Germany

11 Department of Environmental Chemistry, IDAEA-CSIC, Jordi Girona 18-26, 08034 Barcelona, Catalonia, Spain

12 Danone Waters, 11 Avenue du Général Dupas, 74500 Evian les Bains, France

13 TZW: DVGW-Technologiezentrum Wasser (German Water Centre), Karlsruher Straße 84, 76139 Karlsruhe, Germany 


$\begin{array}{ll}\text { Abbreviations } \\ \text { ABS } & \text { Acrylonitrile butadiene styrene } \\ \text { AFM } & \text { Atomic force microscopy } \\ \text { ATR } & \text { Attenuated total reflection } \\ \text { FPA } & \text { Focal plane array } \\ \text { FTIR } & \text { Fourier transform infrared } \\ \text { HQI } & \text { Hit quality index } \\ \text { ICA } & \text { Independent component analysis } \\ \text { ILC } & \text { Interlaboratory comparison } \\ \text { IR } & \text { Infrared } \\ \text { JRC } & \text { Joint Research Centre } \\ \text { LDIR } & \text { Laser direct infrared imaging } \\ \text { LOD } & \text { Limit of detection } \\ \text { LOQ } & \text { Limit of quantification } \\ \text { MCR-ALS } & \text { Multivariate curve resolution-alternating } \\ & \text { least squares } \\ \text { H-FTIR } & \text { Micro-FTIR } \\ \text { MP(s) } & \text { Microplastic(s) } \\ \text { OT } & \text { Optical tweezers } \\ \text { PA } & \text { Polyamide } \\ \text { PC } & \text { Polycarbonate } \\ \text { PE } & \text { Polyethylene } \\ \text { PET } & \text { Poly(ethylene terephthalate) } \\ \text { PMMA } & \text { Poly(methyl methacrylate) } \\ \text { PP } & \text { Polypropylene } \\ \text { PU } & \text { Polyurethane } \\ \text { PS } & \text { Polystyrene } \\ \text { PT } & \text { Proficiency tests } \\ \text { PTFE } & \text { Polytetrafluoroethylene } \\ \text { PVC } & \text { Poly(vinyl chloride) } \\ \text { QA } & \text { Quality assurance } \\ \text { QC } & \text { Quality control } \\ \text { QCL-IR } & \text { Quantum cascade laser-based infrared } \\ \text { RDF } & \text { Random decision forest } \\ \text { RM } & \text { Micro-Raman spectroscopy } \\ \text { SEM } & \text { Scanning electron microscopy } \\ \text { SERS } & \text { Surface-enhanced Raman spectroscopy } \\ \text { TERS } & \text { Tip-enhanced Raman spectroscopy } \\ \text { WHO } & \text { World Health Organization } \\ & \end{array}$

\section{Introduction}

Microplastics (MPs) are considered as a new class of contaminant whose emergence derives from the enormous growth in polymer use over the last 70 years and the progressive fragmentation of the resulting plastic waste debris dispersed into the environment $[1,2]$. There is an ongoing discussion about the definition and categorization for the term "microplastics" $[3,4]$. The Technical Report CEN ISO/TR 21960 defines "microplastic" as "any solid plastic particle insoluble in water with any dimension between $1 \mu \mathrm{m}$ and 1,000 $\mu \mathrm{m}$ " and "large microplastic" as particles between 1 and $5 \mathrm{~mm}$ [5]. In contrast, the Californian State Water Resources Control Board currently defined "'Microplastics in Drinking Water' [...] as solid polymeric materials to which chemical additives or other substances may have been added, which are particles which have at least three dimensions that are greater than $1 \mathrm{~nm}$ and less than 5,000 micrometers $(\mu \mathrm{m})$. Polymers that are derived in nature that have not been chemically modified (other than by hydrolysis) are excluded [6]." Within this resolution, a size-based nomenclature differentiates between "nanoplastics" ( $1 \mathrm{~nm}$ to $<100 \mathrm{~nm})$, "sub-micron plastics" (100 nm to $<1 \mu \mathrm{m})$, "small microplastics" $(1 \mu \mathrm{m}$ to $<100$ $\mu \mathrm{m})$, "large microplastics" (100 $\mu \mathrm{m}$ to $<5 \mathrm{~mm})$, "mesoplastics" ( $5 \mathrm{~mm}$ to $<2.5 \mathrm{~cm}$ ), and "macroplastics" (> $2.5 \mathrm{~cm}$ ). Another distinction can be made between "primary" and "secondary" MPs. Primary MPs are intentionally manufactured plastic particles added to products for functional purposes, e.g., as abrasive material in toothpaste, exfoliants, and other cosmetics. Secondary MPs originate from larger plastic items which have degraded into smaller fragments (due to UV radiation as well as mechanical, physicochemical, and biotic factors), including MPs that are formed from plastic packaging during use such as abrasion from bottles and caps. These MPs may even break down into nanoplastics [7-11].

With increasing numbers of scientific publications on MP contamination in environmental matrices as well as in food and drinking water, the calls for harmonized methods become louder. In fact, this is one of the research gaps highlighted in the latest report of the World Health Organization (WHO): "a set of standard methods is needed for sampling and analyzing MPs in drinking-water and fresh water." [12]. Moreover, due to the California Safe Drinking Water Act and the Directive (EU) 2020/2184 on the quality of water intended for human consumption, standard methodologies for monitoring MPs in drinking water are needed [13, 14]. To date, many different methods have been reported by numerous laboratories as summarized by Koelmans and colleagues who assessed the quality of MP detection in fifty studies analyzing water from rivers, lakes, groundwater, tap water, bottled drinking water, and wastewater [15]. From these studies, in which MP concentrations varied across ten orders of magnitude $\left(10^{-2}-10^{8}\right.$ particles $/ \mathrm{m}^{3}$ ), Koelmans et al. concluded that $92 \%$ of the reviewed studies were not fully reliable in certain quality assurance (QA) aspects [15]. Furthermore, a huge diversity between the experimental setups of the examined studies was shown. The review by Koelmans and colleagues emphasizes a lack of comparability of the results from different studies, which makes the monitoring and controlling of potential MP release nearly impossible. Instead, as Zarfl summarizes in her review, the "standardization of MP analytical methods on the basis of research aim will help to make study results comparable and obtain a more comprehensive picture of MP abundance and fate in the environment." [16]. One important step toward reproducible and comparable studies was recently made by 
Cowger and colleagues, who reported a detailed guideline on a huge number of steps to be noted during microplastic analysis [17].

Apart from the absence of standardized sampling procedures $[11,12,18,19]$, the analytical difficulties in MP research are further compounded by the limited use or nonavailability of common quality control (QC) measures [19, 20]. In particular, there has been a lack of analytical standards, appropriate reference materials, and interlaboratory comparison (ILC) studies. For the latter of these, the first initiatives were launched only recently [21-25]. The "Discussion Paper: Microplastics Analytics - Sampling, Preparation and Detection Methods" focuses on different physicochemical methods and analytical approaches in order to achieve valid methods and comparable results [3]. Recently, an updated version of the document "Status Report: Analysis of microplastics - Sampling, preparation and detection methods" was presented [26]. The first "Standardized protocol for monitoring microplastics in seawater" was published in 2019 as a result of the "BASEMAN" project [27]. It recommends procedures for MP sampling, processing, and analysis for surface and water column seawater samples, beginning with particles $>100 \mu \mathrm{m}$. Enders et al. published a protocol to extract MPs with sizes between $10 \mu \mathrm{m}$ and $5 \mathrm{~mm}$ from environmental samples [28] and proposed several options to size-fractionate larger from smaller particles. Unfortunately, the proposed operating procedures are not applicable for the analysis of particles in the lower size range (especially below $10 \mu \mathrm{m}$ ).

The most common techniques to reliably identify MPs are spectroscopic (infrared (IR) spectroscopy and micro-Raman spectroscopy (or Raman microspectroscopy, RM)) or thermal degradation methods $[19,29,30]$. While the mass-based thermo-analytical methods can determine the overall mass of different polymer types, the particle-based spectroscopic methods provide information on the polymer type, number, size and size distribution, and morphology separately for all analyzed plastic particles in a sample [3, 11,31].

Furthermore, in considering the applicability of the two approaches, it must be noted that drinking water samples can potentially contain high numbers of small $(<10 \mu \mathrm{m})$ MPs of several polymer types [32-34]. One spherical particle with a diameter of $10 \mu \mathrm{m}$ and a density of $1 \mathrm{~g} / \mathrm{cm}^{3}$ has a mass of 0.5 $\mathrm{ng}$, while one particle with a diameter of $1 \mu \mathrm{m}$ equals a mass of only $0.5 \mathrm{pg}$. Particles of such small size can still be identified individually via spectroscopic methods (RM). In contrast, thermo-analytical methods can only detect them, if they occur in large numbers so that their total mass (one polymer type) exceeds the limit of detection (LOD) of the method (which lies in the range of $\mu \mathrm{g},[26])$.

Other important types of information, especially in relation to toxicological concerns, is the number, size or size distribution, and shape of the MPs. It was, for example, shown that polystyrene (PS) particles below a size of $4 \mu \mathrm{m}$ can pass human intestinal epithelium cells in minor fractions [35]. Spectroscopic methods are particularly well suited for gathering these characteristics, because they permit a direct enumeration of potential MPs and in contrast with colorimetric methods (use of dye) or morphological methods (e.g., scanning electron microscopy, SEM); they are capable of specifically determining their identity through a complete spectral pattern recognition. Given these advantages, this consensus paper focusses on spectroscopic methods as the means to satisfy the analytical requirements for MP analysis in clean water.

To this end, a group of 12 European analytical laboratories and institutions (from Germany, France, Italy, and Spain) experienced in spectroscopic MP analysis has discussed and developed a joint working paper, describing the consensus for sampling procedures and detection methods used in these laboratories. The present paper compiles all the information gathered in working meetings and discussions together with critical review of literature published by other authors and provides a guideline that describes minimum requirements and best practices for MP analysis by spectroscopic methods in clean waters. Clean waters, for the purpose of this consensus paper, comprise all waters with a low content of background matrix, in particular drinking water, bottled and tap waters but also other water types such as clean natural fresh waters or injectable water for medical/pharmaceutical applications, e.g., 1/ Ph. Eur. 2.9.19. [36]. While preparing the manuscript, an extensive literature research was conducted (until 31.03.2021) via the most common databases: ISI Web of Knowledge, Google Scholar, and SciFinder. The search terms included, among others, microplastic, analysis, identification, quantification, characterization, vibrational spectroscopy, Raman, infrared, and drinking water. Then the publications related to guidelines for Raman and IR analysis of microplastics were selected and discussed in this review manuscript.

In this paper, all relevant methodological steps toward a valid and reliable MP identification are described and discussed. It includes precautions and advice on sampling and sample preparation, avoidance of sample contamination, measurement methods, data processing, and method validation. In addition to their discussion in the text, all these points are summarized in Table 1. The minimum requirements and the best practice approaches are designed to (i) improve the reliability of MP analysis by helping labs to identify pitfalls in the analytical method and, thus, to avoid generating falsepositive and false-negative results; (ii) facilitate and improve the planning of MP analysis; and (iii) provide a better understanding regarding the evaluation of already existing studies. Overall, the present review attempts to make an important step toward harmonization of MP analysis in clean waters in order to allow the comparison of results obtained in different studies by using similar or harmonized methods. These proposals and 
Table 1 Minimum requirements and the best practice guidelines for the analysis of microplastics in drinking water and other clean water samples with micro-Raman and micro-infrared spectroscopy

Method Minimum requirement $\quad$ Best practice

Avoiding sample contamination

Air purity, type of floor/wall

Type of extraction hood

Type of lab coat, clothes, gloves

Operator precautions

Sampling

Type of sampling container or online process

Volume of sample

Number of replicates

Preparation of sampling container

Sample preparation

Cleaning of the outer side of the sampling container

Addition of reagent, use of tools

Sample filtration

Filtration steps

Nature of filter

Free choice

Filter features (pore size, dimensions) Filter pore size must be adapted to the size of particles delivered in the report (information about filter pore size should be given in the report)

Nature of filter holder and other materials used

Avoid plastic tools as alternatives are existing Use of plastic devices (e.g., PTFE) needs critical and
Clean lab, linoleum or tiling floor, no carpet

Controlled air flow, clean room

Laboratory hood surfaces must be thoroughly cleaned Laminar flow cabinet with filtered liquid (e.g., water) to avoid microparticle contamination

Cotton lab coat, beneath: avoid all clothes with potential release of synthetic textile fibers

Gloves: if used, check critically for potential contamination

Wash hands, tie back hair, if mask must be worn, use N95

Clean containers

Minimize plastic use during sampling

Volume adapted considering the water type (number and size distribution of microparticles)

\section{1}

Mechanical cleaning and rinsing (e.g., cleaning with particle-free water)

Mechanical cleaning and rinsing before entering the lab hood/clean bench

If adding reagents: check for purity/possible contamination through blank samples; filtration advised with further check of contamination

Use of pre-cleaned glassware and tools (glass pipette)

Avoid plastic tools/pipettes

1 (e.g., cellulose acetate, $0.2 \mu \mathrm{m}$ )

Option to dissolve minerals: $\mathrm{Na}_{4}$ EDTA solution ( 250 $\mathrm{g} / \mathrm{l})$ depending on the amount of calcium and magnesium in the sample prepared from $\mathrm{Na}_{4}$ EDTA $>99 \%$, filtered through a syringe membrane filter

Optional: fractionated filtration, for example, with multiple filters of different pore sizes (same material or any other approved material)

Check the quality of the filter surface: it should be default-free and very flat

For automated systems:

- Silicon (FTIR, Raman)

- Metal (Au, Al)-coated PC filter (IR, Raman): exclusion of PC results advised; at least careful checking of the level and stability of the blank needed

- Aluminum oxide (IR)

- PTFE (Raman): exclusion of PTFE results advised; at least careful checking of the level and stability of the blank needed

For non-automated systems:

Free choice, e.g., above-named filters or others, e.g., nitrocellulose

To reduce the time of analysis, the smallest possible filter surface should be chosen

If background correction is applied in spectroscopy, pay attention to the distances between pores to obtain adequate background signals from the filter very strict control (check blanks) with possible exclusion of corresponding polymer particles

Stainless steel, glass, or colored PTFE (colored with blue or red dye; allows the laboratory to give results for PTFE, with exclusion of the dyed material from the results) 
Table 1 (continued)

\begin{tabular}{lcc}
\hline Method & Minimum requirement & Best practice \\
\hline Volume of sample filtered & $\begin{array}{c}\text { Sub-filtration possible; if it is done, it has to be stated in } \\
\text { the report }\end{array}$ & $\begin{array}{l}\text { Ideally filtration of the entire sampling volume to } \\
\text { reduce inhomogeneity when aliquoting the sample } \\
\text { Glassware rinsed once with particle-free water after } \\
\text { initial filtration of the sample to maximize the recu- } \\
\text { peration of microparticles potentially stuck at the } \\
\text { surface of glassware. Be aware that the rinsing step } \\
\text { might bring contamination (check blank values) }\end{array}$
\end{tabular}

Handling, transport, and storage of Protection of the filter from atmospheric deposit the filter

Laboratory blanks

Matrix used

Frequency of blank samples during routine analysis

Acceptance criterion for blank routine analysis (MPs/blank), for validation or invalidation of the series

Analysis

Particle detection mode

Size range of MPs targeted $(\mu \mathrm{m})$

Libraries used

Match acceptance criterion between sample spectra and database reference result in a loss of particles) and tested accepting the results of the series some contamination occurred during sample leading to false-positive results the results of this polymer and give additional etc.)

Parameters for image acquisition (e.g., focus, correct size determination) range and distribution) $\mu \mathrm{m},>500 \mu \mathrm{m}$ types) needed, e.g., in glass Petri dishes or metal containers

It is highly recommended to avoid plastic containers (possible contamination; electrostatic charging may

Particle-free water: tested in the lab or water bought

1 blank per series or day for a maximum of 10 samples More than one blank per day or 10 samples

The sum of all kinds of MPs in the blank sample must not exceed the LOD of the method (see below) for

If the number of MPs in the blank sample is higher, processing that could have polluted the samples,

Exceptional procedure: If a high contamination with only one polymer type occurs, the lab can exclude information about the contamination (number, size, contrast/brightness) have to be adapted in order to obtain correct images for particle detection (e.g., for

Information on smallest particle size analyzed (size

If particle numbers are reported in a binned form, the following size classes should be used: $1-5 \mu \mathrm{m}, 5-10$ $\mu \mathrm{m}, 10-20 \mu \mathrm{m}, 20-50 \mu \mathrm{m}, 50-100 \mu \mathrm{m}, 100-500$

Minimal included polymer types: PE, PET, PP, PS, PC, PVC, PMMA, PTFE, nylon (PA), PU (several

Natural materials present in samples (e.g., proteins, cellulose) to avoid mistaking with, e.g., PA (see Supplementary information (ESM), section S1)

If the laboratory is using a fixed limit for automatic acceptance of spectral matching with the database (e.g., hit quality index, HQI):

The minimum limit can be set at a matching result of, for example, $>70 \%$

The lab has to approve initially that the automatic identification for spectral matching above this value is correct, e.g., through operator/human review of the characteristic peaks in the spectra.
Auto or semiautomatic particle detection possible.

Dark-field illumination can be used to improve the detection of small particles $(<5 \mu \mathrm{m})$.

Critical parameters for particle detection may ideally be adjusted automatically or should be fixed in order to avoid inter-operator bias.

Less important for mapping/imaging during Raman/IR measurement.

For future best practice, the specific particle sizes, e.g., in the form of raw data, should be provided for further data analysis and modeling.

Additionally, spectral data for additives (e.g., pigments (Raman)), elastomers, further naturally occurring materials (e.g., minerals)

Homemade spectral database, for example, including materials from sample packaging, containers, and materials used in the laboratory

Pay attention to the spectra of nylon and proteins, which are very similar in IR and Raman spectra (compare "Data processing" and ESM, section S1). The same kind of spectral similarities could issue with Polyethylene and molecules containing long $\mathrm{CH}$-chains, e.g., stearates leading to potential false-positive identification. 
Table 1 (continued)

$\begin{array}{lll}\text { Method } & \text { Minimum requirement practice }\end{array}$

Objective used

IR acquisition mode

Range of acquisition $\left(\mathrm{cm}^{-1}\right)$

Raman laser wavelength used (nm)

Raman laser beam spot size $(\mu \mathrm{m})$

Raman laser parameters

Spectral resolution $\left(\mathrm{cm}^{-1}\right)$

Number of particles/surface of filter analyzed
Afterwards, the lab is free to consider particles with spectral matching below this value as identified, when the identity is confirmed via operator/human review.

Be aware that different software may produce different values for the HQI and that a verification of the algorithm has to be done to confirm the correct identification of the material (see "Data processing").

Besides classical database search, other identification techniques, such as a homemade semiautomatic identification via mathematical algorithms (e.g., classical least squares (CLS), including manual review of results), model-based classification (e.g., random decision forest (RDF) classifiers) are possible after validation of the recognition model.

Depends on samples and equipment type - must allow to obtain a good image/signal

IR: $1250-3600 \mathrm{~cm}^{-1}$

Raman: 200-2000 $\mathrm{cm}^{-1}$

$532 \mathrm{~nm}$ or $785 \mathrm{~nm}$

Spot size depends on the instrumentation (information on the spot size has to be given in the report)

Laser parameters should not cause particle destruction (RM)

Acquisition time minimal $1 \mathrm{~s}$ for single particle measurement to reach an acceptable signal-to-noise ratio. For imaging, shorter time can be used.

IR $: \leq 8 \mathrm{~cm}^{-1}$

Different approaches are possible to analyze particles on the filter. Different possibilities are listed below (beginning with the most favorable model)

(1) If an automatic counting system/imaging for particles is used, the whole surface of the filter should be scanned for the total number of particles. Depending of this number, the following models can be used:

(1 A) THE TOTAL SURFACE MODEL

If the total number of particles is $<1000(<500$ for practical reasons, $<5000$ or 7000 for best practice), all particles on the filter surface should be analyzed by spectral recognition.

If the total number of particles is $>1000$ :
Adjust the objective to the analyzed particle size, e.g., for Raman, particles of $1 \mu \mathrm{m}$ can be measured with a $50 \times$ objective

Transmission and reflection modes for micro-(FT)IR are easier/faster to use

ATR: only for particles $>100 \mu \mathrm{m}$. Slower, more difficult to use and attention must be paid to the cleaning of the device (germanium/diamond) with contact mode to get rid of any cross contamination

IR: entire MID-IR range

Raman: 50-4000 $\mathrm{cm}^{-1}$ (Raman)

Down to $1 \mu \mathrm{m}$, if particles of that size have to be analyzed

Measurement time as long as necessary to get good spectra, but as short as possible to save time.

ADVICE: Work as much as possible in non-destructive mode or with low laser power.

Some examples are given for a mean generic value to start the acquisition testing of a sample:

silicon filter/magnification $20 \times(\mathrm{NA} 0.50)$ or $50 \times(\mathrm{NA}$ $0.55) /$ laser power $(532 \mathrm{~nm}) 5 \mathrm{~mW}$ or $6 \mathrm{~mW}$, (785 nm) $15 \mathrm{~mW}$

Au-coated PC filter/magnification 20× (NA 0.50) or $50 \times(\mathrm{NA} 0.55) /$ laser power $(532 \mathrm{~nm}) 3 \mathrm{~mW} / 5 \mathrm{~s}$, $10 \mathrm{~s}$ or $20 \mathrm{~s}$

Al-coated PC filter/magnification 50× (NA 0.55)/laser power $(532 \mathrm{~nm}) 3 \mathrm{~mW}-(785 \mathrm{~nm}) 5 \mathrm{~mW} / 2 \mathrm{~s}$

Spectral recognition of all particles on the filter, if possible.

If too many particles occur on the filter, a representative aliquot can be taken to decrease the total number of particles (see "Models for sub-sampling of particles deposited on a filter"). 
Table 1 (continued)

$\begin{array}{lll}\text { Method } & \text { Minimum requirement practice }\end{array}$

\begin{tabular}{|c|}
\hline $\begin{array}{l}\text { ALL particles }>50 \mu \mathrm{m} \text { (up to } 1000 \text { ) have to be counted } \\
\text { and measured (if possible with imaging/sizing } \\
\text { system), especially for environmental or complex } \\
\text { (e.g., food) samples. For particles }<50 \mu \mathrm{m} \text {, one of } \\
\text { the following models should be chosen: } \\
\text { (1 B) THE RANDOM MODEL } \\
\text { Choose randomly a selected number ( } 1000 \text { particles at } \\
\text { minimum, } 500 \text { for practical reasons, } 5000 \text { or } 7000 \\
\text { particles for best practice) to be analyzed/identified. } \\
\text { (1 C) THE "CAKE" MODEL } \\
\text { If random particle selection is not possible, at least one } \\
\text { region representing "a piece of cake" (from the } \\
\text { center of the filter to the border of the filter, for } \\
\text { example, a quarter of the filter is a piece of cake by } \\
1 / 4) \text { has to be chosen for analysis. Its surface should } \\
\text { be at least } 20 \% \text { of the total filter surface, when } \\
\text { analyzing particles down to } 10 \mu \mathrm{m} \text { (IR) or } 5 \mu \mathrm{m} \\
\text { (RM), and at least } 4 \% \text { of the total filter surface, when } \\
\text { analyzing particles down to } 1 \mu \mathrm{m} \text {. Additionally, the } \\
\text { number of particles analyzed on this piece of cake } \\
\text { must exceed } 1000 \text { (500 for practical reasons, } 5000 \text { or } \\
7000 \text { particles for best practice). } \\
\text { (1 D) THE HELIX or "SNAIL" MODEL } \\
\text { If it is technically not possible to choose random } \\
\text { model, at least } 5 \text { regions on the filter have to be } \\
\text { chosen for analysis. Their total surface should be at } \\
\text { least } 20 \% \text { of the total filter surface, when analyzing } \\
\text { particles down to } 10 \mu \mathrm{m} \text { (IR) or } 5 \mu \mathrm{m} \text { (RM), and at } \\
\text { least } 4 \% \text { of the total filter surface, when analyzing } \\
\text { particles down to } 1 \mu \text { m. Additionally, the number of } \\
\text { particles analyzed for the chosen regions must ex- } \\
\text { ceed } 1000 \text { ( } 500 \text { for practical reasons, } 5000 \text { or } 7000 \\
\text { particles for best practice). }\end{array}$ \\
\hline
\end{tabular}

(2) If there is no automatic counting system/imaging for particles

- All particles on the entire filter surface must be analyzed with spectral recognition up to a total number of particles of 1000 (500 for practical reasons) as minimum requirement ( $>2000$ for best practice approach)

- If the total number of particles is $>1000$, use a combination of:

Analysis of all particles $>50 \mu \mathrm{m}$ up to 1000 (500 for practical reasons) (possible with imaging/sizing system) AND selection of one statistical model from B to $\mathrm{D}$ for particles $<50 \mu \mathrm{m}$.

The final results are obtained as the sum of the particles above and below $50 \mu \mathrm{m}$ (measured with one of the above-described models; each extrapolated to all

Method validation particles detected in the corresponding size range).

Description of the way to validate the method
I. Verification of size measurement of the equipment, e.g., with particles of known size

II. Verification of qualitative polymer identification at the claimed minimal size, at least for the main polymer types (PE, PET, PC, PP, PVC, PS, etc.)

III. Determination of the LOD as the mean of all MPs identified in 10 blank samples + their threefold standard deviation: $L O D=$ mean $_{10}$ blanks $+3 * s_{10}$ blanks Re-determination after modifications of the method
IV. Verification of the recovery rates of the entire method

V. Inter laboratory comparison (ILC) and proficiency tests (PT) 
Table 1 (continued)

\begin{tabular}{|c|c|c|}
\hline Method & Minimum requirement & Best practice \\
\hline \multicolumn{3}{|l|}{ Data processing } \\
\hline Information to be given in the report & $\begin{array}{l}\text { - Total number of particles in the sample or sub-sample } \\
\text { (if available) } \\
\text { - Number of particles analyzed } \\
\text { - LOD } \\
\text { - Number of total microplastics identified (calculation } \\
\text { or measurement) } \\
>\text { By type of polymer } \\
>\text { By size ranges } \\
>\text { No blank subtraction } \\
\text { - If sub-sampling during measurement was done: ana- } \\
\text { lyzed area of the filter (\%) or statistical percentage of } \\
\text { analyzed particles on the total number of particles } \\
\text { (\%) } \\
\text { The laboratory should report quantitative results only, } \\
\text { if the results exceed the LOD. Otherwise, results can } \\
\text { be given as < LOD. }\end{array}$ & $\begin{array}{l}\text { Additional information, if requested: } \\
\text { - Shape (fragment, fiber, or bead) } \\
\text { - Color, pictures of particles or filters } \\
\text { - Number of (potential) MPs not included in the mini- } \\
\text { mum set of polymer classes. Total number of } \\
\text { non-plastic particles identified (e.g., minerals, } \\
\text { proteins, cellulose), by type, by size range, etc. } \\
\text { - Total number of non-identified particles } \\
\text { If qualitative information is given about particles } \\
\text { smaller than the pore size of the filter, it must be } \\
\text { demonstrated that these particles were intrinsic to the } \\
\text { sample. Further, it must be stated that these data are } \\
\text { not representative. } \\
\text { For future best practice, the specific particles size, e.g., } \\
\text { in the form of raw data, should be provided for } \\
\text { further data analysis and modeling. }\end{array}$ \\
\hline
\end{tabular}

best practice approaches are intended to support the different standardization processes that are ongoing throughout national and international normalization committees.

\section{Measures to avoid sample contamination}

The laboratory working environment is a critical point for analysis of MPs. Koelmans and colleagues [15] pointed out that laboratory working conditions represent a key factor in improving the quality and comparability of results. Some recommendations have already been described to improve the quality of analytical methods [3, 12, 20, 37]. These include the avoidance of plastic items and synthetic components during the entire analytical process from sampling to analysis. Figure 1 gives an overview of critical points concerning a quality-controlled microplastic analysis.

As a starting point, the laboratory should be largely free of MP particles or fibers. In order to ensure this, the floor of the laboratory needs to be of an easily washable material and must be regularly cleaned. Some laboratories use sticky anticontamination mats at the entrance [20]. Airborne particles and fibers are one of the major causes of external sample contamination [38] and must be assessed and minimized by appropriate strategies. As described in previous studies, the use of controlled air flow is recommended to maximize air purity and minimize airborne contamination [15, 33]. Sample handling should be performed in a "clean air laboratory" [39-41] or in a laminar flow cabinet [3, 42]. By choosing a laminar flow cabinet, at least ISO Class 5 (3520 particles $/ \mathrm{m}^{3}$ for particles $\geq 0.5 \mu \mathrm{m}$ ) is advisable, whereas a cabinet of ISO Class 3 (35 particles $/ \mathrm{m}^{3}$ for particles $\geq 0.5 \mu \mathrm{m}$ ) [43] is preferable, especially for the analysis of MPs in the lower $\mu$ - range. Additionally, surfaces in the lab and the laminar flow cabinet must be cleaned regularly [20].

Due to the risk of contamination by plastic microfibers, clothing requires special attention [37]. In all handling steps, cotton or antistatic lab coats (particle free) must be worn by all analytical staff. Moreover, clothes containing synthetic fibers, even when worn underneath a lab coat, may increase the ambient level of MPs [19]. General precautions during sample handling have to be followed by operators in order to decrease the risk of contamination (e.g., wash hands, tie back long hair, no make-up, nail polish nor hydration cream), as these products may contain and release MPs [44].

Wherever safety considerations permit, the use of disposable laboratory gloves should be avoided, as they might cause sample contamination. If necessary, the usage of gloves must be critically checked by analyzing blank samples [45]. Moreover, recent pandemic events may impose the need for laboratory staff to use temporarily specific personal protection equipment as (single use) masks. These masks can be made from polypropylene (PP) and thus might release numerous PP microparticles into the lab environment [46]. In general, labs are encouraged to critically reflect on any temporary modification of usual working practices that could interfere with MP measurements.

Cleanliness of the working area needs to be evaluated by the operators. Schymanski et al. performed a periodical evaluation of particle levels with a particulate measuring device inside the hoods to verify their efficiency [33]. Some authors deposit empty filters or petri dishes next to the working area to check for background contamination levels [47, 48].

To evaluate the contamination level in parallel with every sample batch, a blank test with particle-free water (recognized quality, e.g., ultrapure, ultra-filtrated, or pyrogenic water) should be performed (see "Laboratory blanks"). 
Fig. 1 Important precautions and advice for the analysis of microplastics

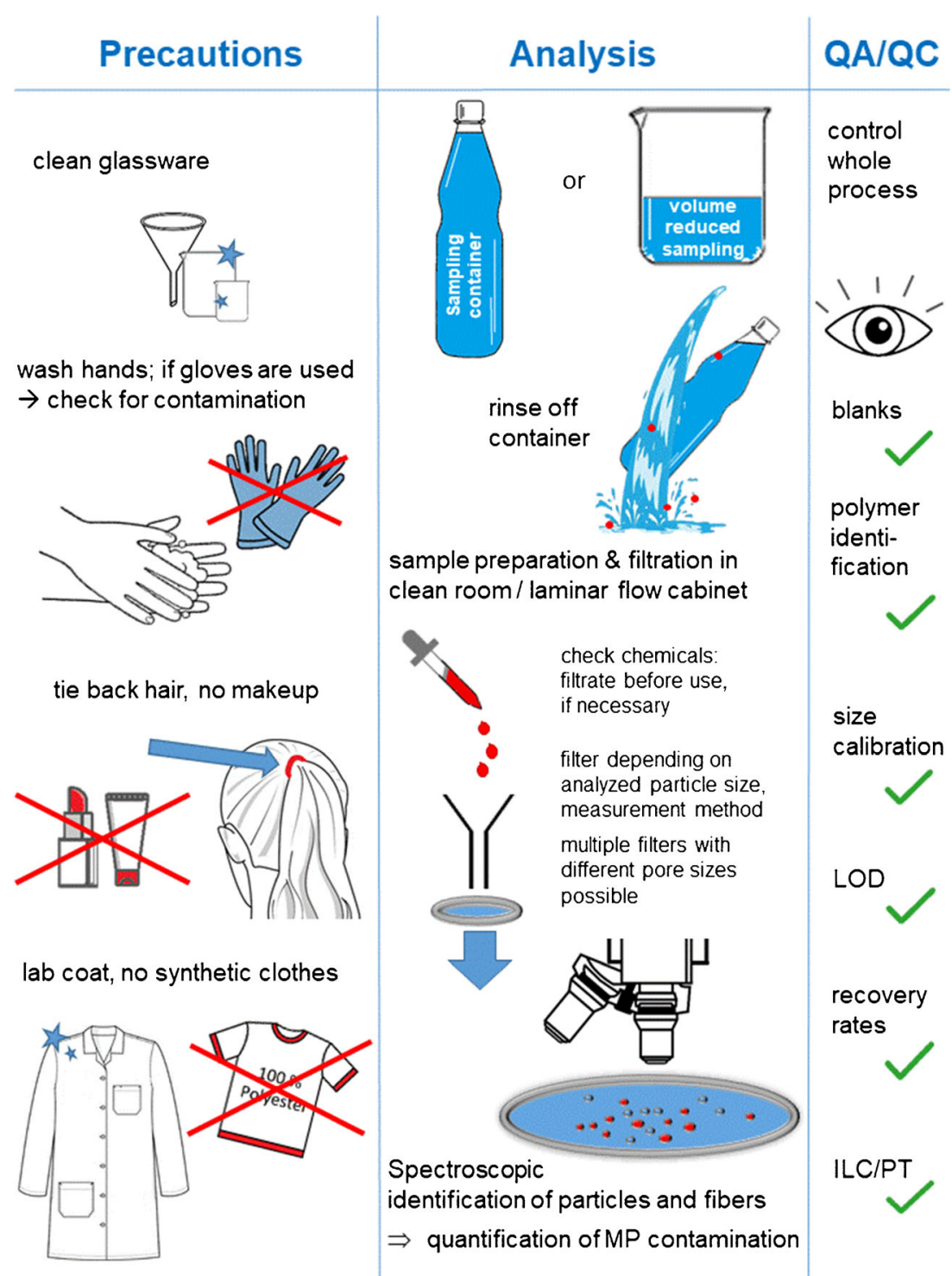

Plastics may only be used if the corresponding polymer is excluded from the results or if it is marked, for example, with a fluorescent dye and can thus be distinguished from sampled MPs. For instance, some laboratories use filtration devices that contain parts made from specifically colored polytetrafluoroethylene (PTFE), which enable the recognition of other PTFE particles that may originate from the sample [49]. If possible, only glass or stainless steel should come into direct contact with the sample. In addition, it is essential that containers for sampling are thoroughly cleaned prior to use [15]. This can be achieved by mechanical cleaning and rinsing with particle-free water. In order to remove particles firmly stuck to container walls, this process may not suffice. Further options for particle removal are baking of containers in a muffle oven [50], sonication of containers filled with water [34], and rinsing with pre-filtered surfactants or chemical solutions.
Potential sample contamination during sampling should be monitored by the analysis of blank samples (see "Laboratory blanks") [15, 20].

Experiments showed that 20 to 80 MPs down to a size of $10 \mu \mathrm{m}$ are present on the external surface of a bottle/ container and could be removed by rinsing the outer surface in order to preserve the bench and the hands of manipulator from this contamination (unpublished data by $\mathrm{N}$. Benismail). Therefore, it is necessary to clean the outside of the container, e.g., with particle-free water and a detergent. Furthermore, within this step, labels should be removed from bottled water whenever possible. A final rinsing with particle-free water should be performed before placing the sample containers in the laminar flow box, where the sample containers are left to dry prior to further handling [32, 33]. 
In order to avoid sample contamination by adding chemicals, it is highly recommended that any particles present in the reagent solutions are removed by pre-filtering the chemicals [20, 51]. This can, e.g., be done with syringe membrane filters (with $0.2 \mu \mathrm{m}$ pore size, e.g., made of cellulose acetate [32]). Instead of syringe membrane filters, the chemicals can be filtered through a polycarbonate (PC) filter (with a pore size of $1.2 \mu \mathrm{m}$ ) as proposed by Weber et al. [52]. Alternatively, $1.2 \mu \mathrm{m}$ and $0.7 \mu \mathrm{m}$ glass-fiber filters can be applied for the filtration of the reagents before use, as reported by Johnson et al. and Kirstein et al. [53, 54], respectively. But, it has to be pointed out that the pre-filtering of chemicals can lead to a contamination with MPs, e.g., particles can be released from the filter membrane, the plastic housing of the membrane filters [55] or from frits of classical filtration devices (unpublished data of B. E. Oßmann). Therefore, both filtered and non-filtered chemicals should be tested for particles in order to decide on a case-by-case basis if filtering is suitable or not.

After filtration, during the transfer to the analytical device and for storage, the filter has to be covered to avoid contamination from the air. Different container types can be used, such as glass petri dishes or metal containers. It is highly recommended that the use of plastic materials (e.g., polystyrene (PS)/PC petri dishes) is avoided.

\section{Sampling}

In the case of MP analysis, samples typically contain a heterogeneous mixture of particles, of which only a very small percentage is actually MPs. Therefore, it is extremely important to analyze this heterogeneity and apply appropriate sampling, as well as sample preparation tools and methods, which shift the system toward a state that guarantees a representative analysis of the lot [56]. Because particles in the size range of $1 \mu \mathrm{m}$ to $1 \mathrm{~mm}$ have a very small mass, it is advisable not to prematurely focus on sample mass, but rather on particle numbers.

Sampling strategies for microplastic analytics in water vary a lot between different water types. Bottled waters are usually sampled as entire bottles/packages and brought to the lab as they are $[32,33,57]$. In contrast, for tap waters, a defined volume is taken with sampling containers (e.g., glass bottles $[34,58])$ or the volume is reduced on-site with special filter cartridges $[53,54,59,60]$. In order to provide representative results, it is important to adapt the sampling volume to the anticipated properties of the sample [20]. In waters, which are expected to have low MP concentrations (e.g., tap water), a much higher sampling volume is required in order to produce representative results, than in waters with higher anticipated MP concentration [15]. The number of (plastic) particles in the sample increases by orders of magnitude with decreasing particle size. Therefore, usually a lower sample volume is required for representative sampling of particles in the smaller micrometer size range. Different research groups, who analyzed MPs $>25 \mu \mathrm{m},>20 \mu \mathrm{m},>6.6 \mu \mathrm{m}$ resp. and $>5 \mu \mathrm{m}$ in tap waters sampled several hundred to several thousand liters $[53,54,59,60]$, while Pivokonsky et al., who analyzed MPs $>1 \mu \mathrm{m}$ in tap water sampled only one liter [34]. If achievable, ideally a triplicate of each sample should be taken and analyzed (best practice) [20].

\section{Sample preparation and filtration}

Particles are usually separated from the water matrix via filtration, either on-site during sampling (see "Sampling") or in the laboratory. In the first case, the filter residue is transferred from the cartridge to another filter in the laboratory; in the second case, samples are filtered through a filter, which is suitable for analysis. The applicability of a filter material depends on the analytical technique that will be used and the particle size to be analyzed.

In general, filters suitable for analysis have to be flawless and flat in order to gather all particles of a similar size within one focus [61]. Filter flatness can be enhanced by using special membrane-flattening holders during measurement [62-64].

The pore size has to be smaller than the minimal particle size, which is reported quantitatively. However, pore size may not be chosen far below the targeted particle size, as a larger pore size simplifies and speeds up filtration and prevents the filter from being clogged or covered with matrix residues [51, 65]. What is more, the filter material must allow optical particle recognition (for visual analysis and methods based on image analysis) and must not interfere during measurement $[61,66]$.

For RM, either silicon membranes or metal-coated (Au or $\mathrm{Al}$ ) PC filters are recommended. Both filter types obtain high contrast to particles, if dark-field illumination is applied [33, $61,66]$. Besides, PTFE membranes are suitable, when used in bright-field illumination [60]. If particles are selected and analyzed manually, other filter materials like nitrocellulose are also adequate $[67,68]$. Gold- and aluminum-coated PC membranes do not interfere with particle spectra as they do not show an own intense Raman spectrum [33, 61]. In contrast, other filters, such as PTFE, silicon, or cellulose, show characteristic peaks themselves. While the spectra of PTFE and silicon can easily be distinguished from targeted plastics, cellulosic filters can interfere during MP identification $[60,61,66]$.

Using IR spectroscopies, one has to differentiate between reflection and transmission measurements. For reflection measurements, gold-coated PC membranes have been applied successfully [69, 70], as well as reflective microscope slides [71]. Further, other light-reflecting surfaces like filters and microscope slides coated with silver or other metals are conceivable 
$[30,70]$. For transmission measurements, substrates must be transparent for IR light in the relevant spectral range. Aluminum oxide membranes (Anodisc) [72], zinc selenide windows [73], or silicon filters [66] are commonly used materials. Anodisc membranes are only infrared transparent between 3600 and $1250 \mathrm{~cm}^{-1}$, which partially masks the IR fingerprint region (1400-600 $\mathrm{cm}^{-1}$ ). Nevertheless, most polymer types can be identified $[63,72,74,75]$. In contrast, silicon filters, which are transparent for the broad mid-infrared range (4000-600 $\mathrm{cm}^{-1}$ ), allow measurement of the IR fingerprint region, while being available in a variety of pore sizes in the micron range. This facilitates sample preparation, while providing a very flat surface. However, custom-made filtration units are necessary as these membranes are typically square-shaped [66].

Some filter materials consist (partly) of plastics (e.g., PTFE, metal-coated PC membranes, and Anodisc with PP support ring), which might lead to sample contamination. When using such filters, it is necessary to critically check this potential contamination route via the analysis of blank samples (see "Laboratory blanks"). If in doubt, it is advisable to exclude this polymer type from MP results to avoid misinterpretation [33].

The sample volume (see "Sampling") filtered for the actual analysis must allow representative analysis and at the same time prevent overloading or clogging of the filter. In order to achieve these goals, one can use different options. Reduction or sub-sampling of the initial sampling volume should be avoided whenever possible, as this will introduce a larger error margin. If this is not possible, information about sub-filtration has to be reported. E.g., for bottled water, it is advisable to filter the entire bottle volume, but when analyzing MPs down to $1 \mu \mathrm{m}$, the filtration volume has to be reduced to get a measurable particle density on the filter [32].

Besides sub-sampling or reduction of the filtration volume, it is possible to use fractionated filtration with filters of different pore sizes to reduce particle numbers and avoid filter clogging. Subsequently, all the filters have to be analyzed separately for MP of different size categories [34, 49, 60]. If samples contain a high amount of background matrix, it may be necessary to add chemicals to the samples prior to filtration or to treat the residue of volume-reduced sampling before transfer to the filters for analysis. Alternatively, one might treat the residues deposited on the filter with chemicals after filtration. For example, Mintenig et al. added hydrochloric acid to dissolve calcium carbonate and iron precipitates (e.g., iron oxides) after filtration [59], whereas Pivokonsky et al. used wet peroxide oxidation to digest organic material in tap water samples [34]. Another option to dissolve carbonate particles is via complexing calcium and magnesium cations by adding an equimolar amount of ethylene diamine tetraacetic acid tetrasodium salt as solution [32]. In order to reduce the risk of sample contamination by the addition of chemicals, such sample treatment steps should only be done if necessary.
The actual filtration area should be reduced to a minimum to decrease measurement time. At the same time, one has to ensure that particles do not overlay, inhibiting correct particle analysis. For example, Schymanski et al. applied a filtration area of $12.6 \mathrm{~mm}^{2}$ (pore size $3 \mu \mathrm{m}$ ) to filter up to $1.51 \mathrm{of}$ mineral water, whereas $\mathrm{O} ß \mathrm{mann}$ et al. applied a filtration area of $\sim 113 \mathrm{~mm}^{2}$ (pore size $0.45 \mu \mathrm{m}$ ) to filter $250 \mathrm{ml}$ of mineral water $[32,33]$. Before filtering the sample, homogenization should be achieved, e.g., by gentle shaking. After filtration, the sample container and the funnel of the filtration device can be rinsed with particle-free water or solvents to recover particles adhering on the walls.

\section{Laboratory blanks}

Careful attention is required to ensure that samples are not contaminated by external particles $[15,33,38]$. For QC and in order to quantify potential external contamination, reagents and materials used for performing sample analysis (e.g., water and chemicals used for rinsing and cleaning of utensils and containers), as well as the entire procedure from sampling to analysis must be controlled [20].

Therefore, particle-free water must be analyzed regularly as a process blank. This blank sample must undergo the same procedure as the actual samples, including all sample preparation steps [76]. For a small series with less than ten samples that are all processed within one day, a single (process) blank sample is sufficient. For large series with more than ten samples, multiple (process) blank samples should be analyzed alongside the sample series (e.g., at least one process blank sample per five or ten samples) [26].

The number of MPs (as a sum of polymers) found in the process blank sample indicates the level of external contamination that has reached the samples during the daily sample manipulation. To validate a series of samples, the particle count from the blank should be compared to the values obtained during the validation stage of the method (see "Method validation and quality controls"). If the result from the daily process blank sample is lower than or equal to the LOD determined during the initial validation of the method, the daily sample manipulation can be considered to have been correctly done in agreement with the given recommendations of this paper. On the contrary, if the number of MPs in the process blank sample is higher than the LOD, the series of samples has to be invalidated. Even when all recommended precautions are taken, unexpected external contamination may still occur in the process blank sample and thus may also have reached the samples.

In the case where the number of MPs detected in the blank value is higher than the LOD, but this value is attributable to a high number of MPs of one polymer type only (with the sum of the others being below the LOD), the results for all other 
polymer types of the sample series may be considered as valid. The conspicuous polymer type must be excluded from all results for this sample series. This procedure should only be used as an exception. Furthermore, as much information as possible should be provided on the contamination (e.g., particle number, size, conspicuous polymer type, potential contamination source).

To insure that the lab is reporting correct values, it is strongly recommended that sample results are presented without prior subtraction of blank values. The LOD of the lab should be expressed each time results are given. When sample results are below the LOD (see "Method validation and quality controls" and "Ways of reporting results and valuable information"), these results should be expressed in the report as $<$ LOD.

\section{Analysis}

\section{(Fourier transform) infrared (FT)IR spectroscopy}

The key parameters that can be adjusted for each (FT)IR instrument are the spectral range; the spectral resolution, i.e., the ability to resolve features within the chosen spectral range; and the number of accumulated sample spectra. Optimizing these parameters on any given instrument is crucial to obtain high-quality spectra [72]. In many cases, a spectral range from 3800 to $900 \mathrm{~cm}^{-1}$ at a resolution of $8 \mathrm{~cm}^{-1}$ with 6 to 30 sample scans is applied $[64,72,73]$.

Different approaches can be followed to identify particles by (FT)IR spectroscopy: Clean individual particles can be measured using an attenuated total reflection (ATR) accessory. ATR-FTIR spectroscopy is limited to relatively big particles (approx. $>100 \mu \mathrm{m}$ ) that can be handled individually.

The most common method of applying FTIR spectroscopy to MPs is micro-FTIR ( $\mu$-FTIR) spectroscopy, where a FTIR spectrometer is coupled to an optical microscope. Because of the diffraction limit, FTIR microscopy's spatial resolution is physically limited to approximately $10 \mu \mathrm{m}[64,70,74,77]$. This limit is further influenced by the microscope objective's numerical aperture according to the Rayleigh criterion. $\mu$ FTIR spectroscopy can be performed in reflectance or transmission mode. The choice of mode depends on the substrate (e.g., filter) onto which the MPs have been deposited. Alternatively, the substrate needs to be chosen depending on the method desired or available (see "Sample preparation and filtration").

Reflectance mode, on one hand, is prone to undesired lightscattering effects on the particle surfaces that lower spectral quality $[30,78]$. Light-scattering effects also interfere when analyzing small particles in the so-called transflectance mode. The IR light beam fully transmits the particles being then reflected from the filter surface, resulting in transmission- like spectra [77]. On the other hand, in reflection measurements, when the IR beam is focused on the particle surface, the radiation only penetrates a few micrometers deep into the particle (wavelength-dependent) [69]. This allows an investigation of the particle surface for polymer modifications due to aging effects or influences of sample preparation processes. In addition, reflectance FTIR spectroscopy has been successfully applied for the comparative FTIR and Raman spectroscopic analysis of MPs [70]. Transmission measurements were shown to yield high-quality spectra; however, thick (polymer-dependent, about $>100 \mu \mathrm{m}$ ) or colored particles may lead to total absorption in transmission mode. Consequently, IR bands converge resulting in unidentifiable spectra. Therefore, the most suitable IR technique should be chosen carefully.

Regardless of whether reflection or transmission measurement is used, a background spectrum needs to be recorded. This is necessary to account for noise from IR active substances other than the target MPs such as water vapor, carbon dioxide in the ambient air, or the substrate the sample is deposited on. Silicon membranes, for instance, show weak bands in the mid-infrared range, like the $\mathrm{Si}-\mathrm{O}-\mathrm{Si}$ stretching vibrations at $1108 \mathrm{~cm}^{-1}$ [66]. This background noise can significantly hamper sample analysis, but can easily be corrected. Before each sample measurement, a background spectrum is recorded similar to a sample spectrum on an unused filter or a clean spot on the sample filter or respective substrate. All measurement parameters must be the same as for samples. Furthermore, the number of co-added background scans must be at least as high as the number of sample scans $[54,79]$. The instrument software usually automatically corrects the sample spectrum through subtraction or division of the background spectrum $[77,80]$.

$\mu$-FTIR analysis can be very time-consuming, when measuring hundreds or thousands of particles one by one. Operator input time can be reduced with automated particle detection and FTIR measurement by using appropriate commercial or open-source software (e.g., GEPARD; see "Raman microspectroscopy").

Measurement time can be accelerated significantly by coupling a focal plane array (FPA) detector to the FTIR microscope. These detectors comprise up to $256 \times 256 \mathrm{MCT}$ (mercury cadmium telluride) detector elements. Consequently, FPA detectors allow the simultaneous and therefore rapid acquisition of thousands of IR spectra in parallel. Furthermore, these spectra are spatially resolved, resulting in so-called IR images of well-defined sample areas. In this way, IR images of entire sample filters can be recorded in relatively short times compared to single-point detectors. Thus, for particle-rich samples, this area-based FTIR imaging can be quicker than a particle-by-particle approach and can be performed automatically, using, e.g., open-source software siMPle [79]. Automated FTIR imaging avoids the need for sub-sampling 
of the filter surface, but produces huge amounts of data that need to be analyzed. Furthermore, flatness of substrates is even more important (see above).

A very seldom used, yet noteworthy, technique is $\mu$-ATR or ATR imaging, which combines $\mu$-FTIR spectroscopy, ATR microscope objectives, and FPA detectors. ATR spectra are, in comparison with transmission and reflectance spectra, less prone to noise, light-scattering effects, and total absorption. However, this technique requires the sample to be deposited on a firm surface that does not break when the ATR crystal is pressed on it [63]. Moreover, particles tend to stick on the crystal, which makes any remeasurement of the sample area impossible.

A new technique, quantum cascade laser-based hyperspectral IR spectroscopy (QCL-IR microscopy), has recently been brought to market. This technique is also called laser direct infrared imaging (LDIR). Unlike traditional FTIR imaging systems, QCL-IR imaging systems make a first scan of the whole sample at one particular wavenumber to identify potential regions of interest. The regions of interest are subsequently scanned over a wider wavenumber range to gather enough spectral information to be able to identify the particles. For instance, Primpke et al. scanned environmental samples at $1470 \mathrm{~cm}^{-1}$ to identify potential MP particles [81]. These were subsequently scanned in the region $1800-1184 \mathrm{~cm}^{-1}$ and $1160-1084 \mathrm{~cm}^{-1}$ to identify the targeted polymer types. They compared the QCL-IR system's performance to stateof-the art FTIR imaging and concluded that the results gained using QCL-IR microscopy were in good agreement with those of the reference method, while being about tenfold as fast [81]. QCL-IR, until now, was successfully applied to samples of soil [82, 83], river [84], and brackish waters [85]. However, further research is needed in order to clarify the feasibility and limitations of this technique for the MP analysis in clean water samples.

\section{Raman microspectroscopy}

Raman microspectroscopy (RM) is a non-destructive analytical method based on the effect of inelastic light scattering on molecules. RM (analogous to IR spectroscopy) provides vibrational fingerprint spectra. The coupling of Raman spectroscopy with confocal optical microscopy and the use of a laser in the visible range allow a significantly better spatial resolution down to $1 \mu \mathrm{m}$ and even below (down to approx. $300 \mathrm{~nm}$ ) compared to $\mu$-(FT)IR spectroscopy [31, 86, 87]. RM offers the advantage of being insensitive to water, which allows the analysis of MP in aqueous and wet samples. A major disadvantage of RM for the analysis of MP in real samples is interference due to fluorescence, which can be caused by inorganic (like clay minerals or dust particles), organic (like humic substances) and (micro)biological impurities in the matrix. Therefore, the choice of suitable measurement parameters (laser wavelength and power, photobleaching, and acquisition time, as well as objective magnification and confocal mode) is important to minimize or avoid interference from strong fluorescence and to improve the quality of spectra. In addition, removal of the (in)organic matrix from complex samples (e.g., [28]) leads to a significant increase of the plastic to non-plastic particle ratio. The spectral range for the Raman analysis of MPs has to cover all spectral features of (synthetic) polymers from 50 to $4000 \mathrm{~cm}^{-1}$ (best practice, or from 200 to $2000 \mathrm{~cm}^{-1}$ as minimum requirement). For the proper identification, both the fingerprint area $\left(50 \mathrm{~cm}-1,500 \mathrm{~cm}^{-1}\right)$ and the area including $\mathrm{C}-\mathrm{H}$ stretching modes of alkyls, alkenes, and aromatic compounds $\left(2800 \mathrm{~cm}^{-1}-3200 \mathrm{~cm}^{-1}\right)$ [87] are important. The entire measured spectral region has to be also covered by the databases, applied for the assignment of MP spectra. Thus, a reliable detection, identification, and quantification of MPs can be achieved. Additionally, not only synthetic polymers, but also additives (e.g., pigments), can be identified by means of RM. Furthermore, complementary information about the sample can be obtained by combining RM with $\mu$-FTIR analysis (e.g., identification of pigment(s) and acrylic resin in paint particles [86]).

Raman imaging is usually performed as point-by-point measurements and is thus time-consuming [86], especially when particles smaller than $10 \mu \mathrm{m}$ are to be analyzed. In contrast to an FPA-FTIR approach, Raman imaging is likely to be less effective than particle-by-particle measurement [62].

Since a very high number of particles have to be measured in order to achieve representative results (see "Models for subsampling of particles deposited on a filter"), automation of the RM is necessary. Currently, both commercial and opensource (GEPARD [49] and TUM-ParticleTyper [62]) programs can be used for automated RM analysis. This way, the analysis of the initial optical image provides morphological characteristics and coordinates for all analyzed fragments, while subsequent Raman measurement delivers their chemical identity. From practical experience, the following parameters/ options have proven to be well suited for automated measurements: $532 \mathrm{~nm}$ (in some cases also $785 \mathrm{~nm}$, e.g., fluorescent samples) laser excitation wavelengths, dark-field (in some cases also bright field) illumination, and objectives with longer working distance.

Before spectrum acquisition, Raman systems must be calibrated. Depending on the instrument, this is commonly done on the $520.7 \mathrm{~cm}^{-1}$ peak of a silicon wafer and by zero-order correction of the used grating $[88,89]$. Furthermore, some Raman instruments allow for an automated calibration in the broad spectral range using an internal $\mathrm{Ar} / \mathrm{Hg}$ calibration lamp [90].

\section{Models for sub-sampling of particles deposited on a filter}

There are two ways of sub-sampling: the samples may be split before filtration (see "Sample preparation and prescription"), 


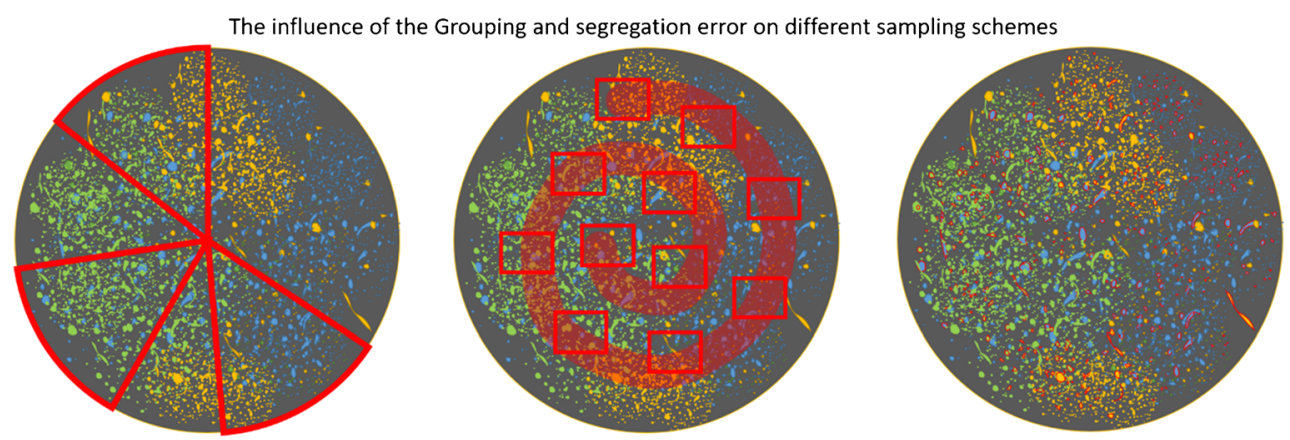

Fig. 2 Illustration of the three sub-sampling models and their effects on introduced errors. Left, "cake" model. Slices are selected for measurement. Depending on their location and size, the grouping of particles may affect the results and introduce errors. Middle, "snail" model. A composite sampling strategy based on the selection of multiple small boxes,

and subsequently, the whole filter is analyzed or alternatively, the whole sample may be filtered and then only a fraction of the filter surface is analyzed.

Total particle numbers (sum of all particles, including minerals, proteins, organic particles, MPs, etc.) in 11 of clean freshwater and drinking water are expected to be up to 10,000 particles, if a lower size limit of $5 \mu \mathrm{m}$ is applied. If the lower size is set to $1 \mu \mathrm{m}$, more than one million particles can be detected (unpublished data of B. E. Oßmann). The subsample size has to be chosen with respect to the total particle number and the expected MP content to obtain representative results (see Fig. 2).

Fig. 3 Dependence of sample size $(n)$ on margin of error $(e=$ $10 \%, 20 \%$ or $30 \%$; e.g., $10 \% \pm$ $0.5 \% \mathrm{MP}, 10 \% \pm 1 \% \mathrm{MP}$, or $10 \%$ $\pm 1.5 \% \mathrm{MP}$, resp.) and total number of fragments $(N)$ for MP contents of $10 \%$ (green), $1 \%$ (blue), and $0.1 \%$ (yellow) for the random model, calculated based on Eq. 1 [87]. Sampling thresholds $(n=2000,5000$, and 7000) are marked in red which should be spread across the filter, so that the edges and middle are represented. Right, random model. Since each fragment is randomly selected, the grouping of particles does not influence their selection. Representativity is only dependant on the number of fragments chosen

When analyzing particles on the filter, it is best to adhere to Gy's theory of sampling, which deals with the errors that are introduced by incorrect sampling specifically for particles [91]. The total sampling error is dependent on two factors, the material heterogeneity and the sampling process [56]. In this context, Thaysen et al. show the spatial pattern of the particles distributed over the filter impacts the different window selection schemes [92].

At this stage, it is advisable to use one of four different models: (1) "total surface/all particles," (2) random, (3) "cake," or (4) "snail” models (see Fig. 2). All models rely

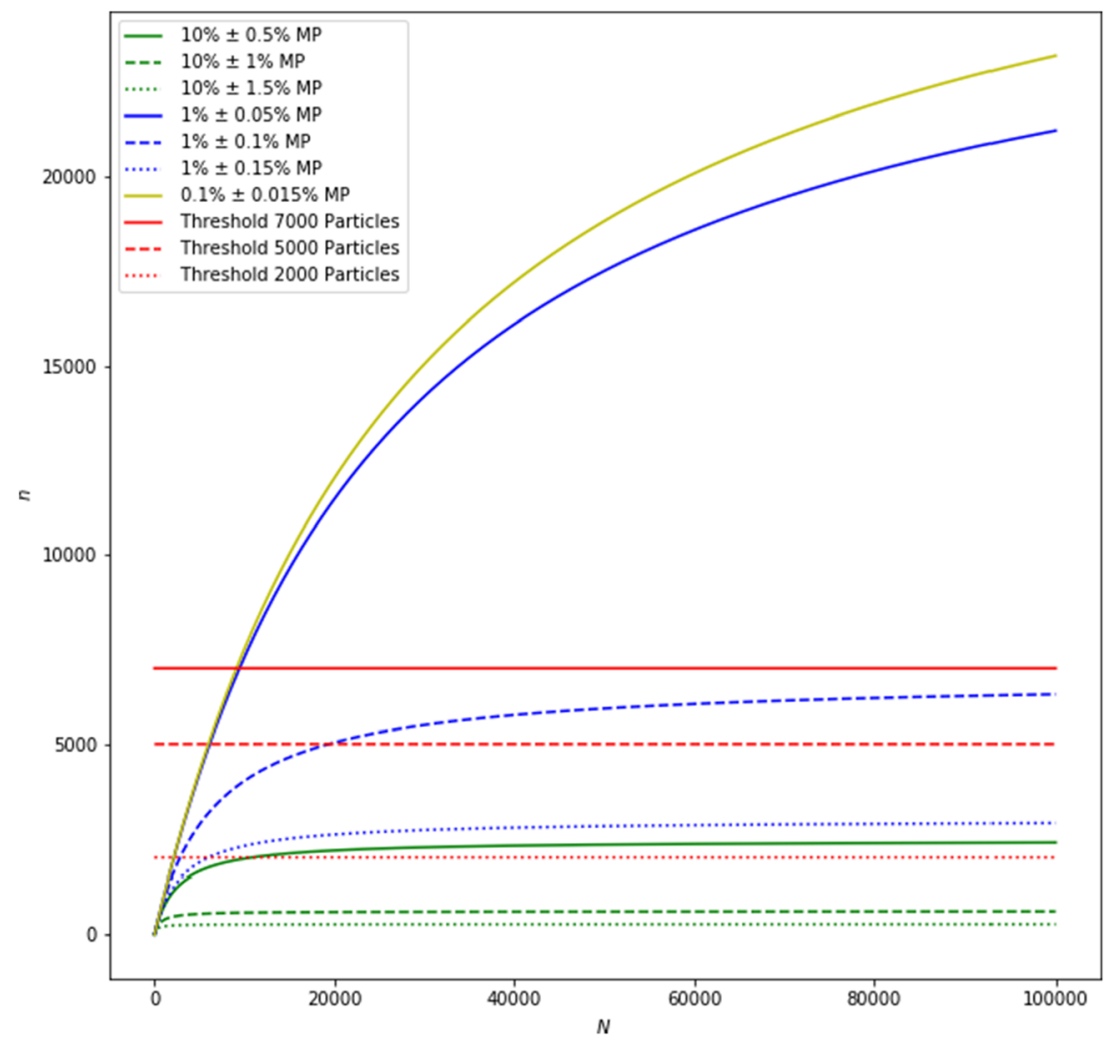


on an automated particle localization routine (open-source or commercial software).

If it is feasible to measure every single fragment (particles and fibers) of the sample, this should be done, as any form of sub-sampling will introduce an error. This model is called "total surface/all particles." For FPA-FTIR imaging, this may be possible for fragments down to $10-20 \mu \mathrm{m}$. For single particle measurement (e.g., for RM), this is appropriate, if the fragment count is below 1000 (or 500 for practical reasons, 7000 best practice). If this number of fragments is exceeded, sub-sampling is necessary to reduce measurement time. This is similar to a second sampling step, where all fragments on the filter represent the lot. The objective is to make a correct selection. According to the theory of sampling [91], all fragments of the lot must have the same probability of being selected as part of the sub-sample. Therefore, no bias is introduced into the process. The investigated fragments are in the size range of 10-500 $\mu \mathrm{m}$ (or 5-500 $\mu \mathrm{m}$ ) and might agglomerate depending on their physical properties. Thus, they may not have an even, random distribution on the filter (Fig. 2: inhomogeneous particle distribution, i.e., grouping of particles according to material type is represented in blue, yellow, and green). This effect is expected to be negligible for fragments at the lower end of the size range, but will have a great effect on fragments at the upper end. A homogenization step for the fragments on the filter would be required. Usually, homogenization implies the reduction of grain size or thorough mixing, none of which is applicable to particles deposited on a filter. Therefore, a virtual mixing is required. The virtual mixing can be achieved by applying three types of sub-sampling. The most advisable sub-sampling strategy is the random model. This corresponds to a random selection of particles, without replacement, from the original lot (all particles on the filter) to form the sub-sample. However, random sampling is not applicable with every software. Another possibility is to take multiple "cake" slices (see Fig. 2). The "cake" model is suitable for taking into account an inhomogeneous distribution of particles between the center and the edge of the filter. In order to represent also inhomogeneous distribution of particles among different filter areas (e.g., filter halves), multiple "cake" slices must be analyzed. To avoid a grouping and segregation error, all large fragments $(>50 \mu \mathrm{m})$ should be measured (up to a number of 1000 or 500 for practical reasons). Particles down to a size of $5 \mu \mathrm{m}$ should be analyzed on "cake" slices that, in sum, cover at least $20 \%$ of the total filter area. If even smaller particles $(1-5 \mu \mathrm{m})$ are targeted, particles in this size range should be analyzed separately, using an objective with higher magnification. In previous works, $4 \%$ of the filter area have been investigated for analyzing this size class [32]. As a suitable alternative, the "snail" model (also called "helix" model, see Fig. 2) can be applied. It uses boxes that are distributed along a spiral to represent all areas of the filter (center and edges). In contrast to the "cake" model, it applies many small boxes. Reducing the box size, while increasing the box number, contributes to a virtual mixing of the fragments and, therefore, reduces the grouping and segregation error [93]. For this model, the same rules for filter area and fragment number apply, as for the "cake" model. The ultimate reduction in box size and increase in box number will finally result in the most advisable model, the random model. The random model can be applied in two forms: a full random sampling (selection of particles regardless of their size) or a stratified random sampling in different size classes (e.g., 1000 particles $\geq 50 \mu \mathrm{m}$ and 7000 particles $<50 \mu \mathrm{m}$ ). The random model ensures an unbiased selection, and the error can be estimated with Eq. 1 based on the final measured MP content [87].

$$
n \geq \frac{P(1-P)}{\frac{e^{2}}{\sigma^{2}}+\frac{P(1-P)}{N}}
$$

\begin{tabular}{lll}
\hline Variable & Symbol & Required information \\
\hline Confidence interval & $\sigma=1.65$ & For 90\% \\
Total number of particles & $N$ & Particle count from detection \\
Estimated MP content & $P$ & From prior experiments/literature \\
Margin of error & $E$ & Inherent to research question \\
Sample size & $n$ & Determined by image analysis \\
\hline
\end{tabular}

The error for different sample sizes and MP contents is displayed in Fig. 3 (the script and an executable file are added in ESM, section S2). The number of particles that need to be chemically identified for an effective quantification depends highly on the expected MP content and the desirable error margin. The goal is to minimize the number of randomly selected particles in the sub-sample to save valuable measurement time. As can be seen in Figure 3, 7000 particles, which correspond to approx. $48 \mathrm{~h}$ measurement at $20 \mathrm{~s}$ acquisition time per particle (e.g., over the weekend, or approx. $12 \mathrm{~h}$ for $5 \mathrm{~s}$ per acquisition time per particle) is suitable for most MP contents and error margins (all green and blue lines except solid blue). If the MP proportion should in fact be smaller than expected (yellow solid line), the measurement of further particles is necessary. This is easy to accomplish as additional particles, which were not measured previously, can be randomly selected from the sample and measured to augment the original sample size. Should the error margin be set too ambitiously (blue solid line) there are two options: (1) measure more particles to be within the error margin or (2) recalculate the error margin and accept a greater error margin than originally desired. In case a very high MP content, e.g., $10 \%$ is expected, a threshold close to 2000 particles may already suffice (all green lines). A threshold of 7000 particles is sufficient 
to ensure a representative sampling in most cases. However, if the MP content is very low (e.g., 0.1\%, see yellow line in Fig. 3 ), only filters with small numbers of deposited particles can be measured quantitatively. This underlines the importance of sample preparation, i.e., removal of inorganic and organic matrices in order to increase the ratio of plastic to nonplastic fragments.

While Gy's theory of sampling and the work of Anger and von der Esch et al. [87] provide means to estimate subsampling errors theoretically, Brandt et al. have investigated the errors introduced by different sub-sampling sizes and models based on actual samples [94]. For that purpose, they have chosen fully analyzed filters from 27 environmental samples (rain water, riverine surface water, riverine sediment or beach sand, wastewater sludge) and performed artificial subsamplings according to the random model (stratified and full random) as introduced here, and different area box-placement strategies, among others, one that resembles the snail model. The environmental samples contained between 1500 and 33,000 particles, and had MP contents between 0.5 and 4.7\%. Brandt et al. [94] found that none of the tested subsampling models clearly outperformed the others, apart from edge scenarios (i.e., area box-placement models perform worse for filters with $<2000$ particles and inhomogeneous particle distribution). Furthermore, they recommended measuring at least 7000 particles or $50 \%$ of the particles using the random models, or $50 \%$ of the filter area in box-placement models, if the sub-sampling error is to be kept below $\pm 20 \%$ (which corresponds to $\mathrm{e}=40 \%$ in the approach of Anger and von der Esch et al. [87]). Concerning the random models, the advice to measure 7000 particles lines out with the recommendations discussed before, but measuring $50 \%$ of the particles on a filter is, in part, stricter than Figure 2 would suggest. However, Brandt et al. [94] found that the standard deviation of the sub-sampling error increases considerably with decreasing measurement fraction, while the graphs in Figure 2 rely on the standard deviation of the normal distribution. Therefore, Brandt et al. tailored their recommendation to meet the $<20 \%$ sub-sampling error requirement with more security. Furthermore, it has to be noted that discussed random subsampling strategy is well applicable for particles larger than $5-10 \mu \mathrm{m}$, where all particles on the filter can be detected, the minimal sample size can be calculated, and errors can be quantified. In contrast, smaller particles might not all be detected on the entire filter, demanding a window-based analysis. For this purpose, a bootstrap method has been recently proposed by Schwaferts et al. [95] to provide an error quantification with confidence intervals from the available window data. In this context, different window selection schemes have been evaluated and there is a clear recommendation to employ random (rather than systematically placed) window locations with many small rather than few larger windows.
In summary, if applicable and technically possible, all particles on the filter should be measured ("total surface/all particles"). Otherwise, a sub-sampling strategy has to be applied. The most recommendable sub-sampling strategy is the random model, because it is not susceptible to inhomogeneous particle distributions on a filter. Only if a random model cannot be realized (e.g., due to technical reasons) the "cake" model or area box-placement models such as the "snail" model should be applied. In that case, sampling of small but numerous boxes or "cake slices" is always preferable.

While for the random model, theoretical error calculations have been proposed, they must still be developed for area boxplacement models (e.g., "cake" and "snail"), which will be especially challenging for heterogeneous samples. Further research is also needed for validating any of these models using actual, fully measured samples and artificial sub-sampling.

\section{Analytical outlook}

In addition to the commonly used IR and Raman approaches previously described, some more recently developed applications/techniques show promising potential for the analysis of micro- and nanoplastic pollution in the future. Theoretically, $\mu$-(FT)IR and RM are limited to minimal particle sizes of about $10 \mu \mathrm{m}$ resp. $300 \mathrm{~nm}$ [31, 64, 77, 86, 87]. Recently, the potential use of confocal RM for particle sizes even below this limit (down to $100 \mathrm{~nm}$ ) was demonstrated [96, 97]. Small micro- and nanoplastic particles (down to a size of $50 \mathrm{~nm}$ ) can be analyzed directly in liquids via a combination of RM and optical tweezers (OT) [98]. Moreover, separation, characterization, and chemical identification of these particles can be realized via online coupling of field-flow fractionation and RM enabled by OT [99]. Furthermore, IR spectroscopy can be combined with other techniques such as atomic force microscopy (AFM) to lower its spatial resolution to about 20 $50 \mathrm{~nm}[100,101]$, thus permitting the analysis of plastic particles at nanoscale. Surface-enhanced Raman spectroscopy (SERS) may play a role in future applications as recently shown by Zhou et al. for PS nanoplastics as small as $50 \mathrm{~nm}$ [102]. The spatial resolution could be improved in the future by the application of tip-enhanced Raman spectroscopy (TERS) [103]. However, the applicability of these methods for different polymer types and on real samples has still to be demonstrated. Especially organic residues, such as humic acids, which might not be entirely removable, might cause interferences [9].

\section{Data processing}

Spectroscopic methods provide information not only on chemical identity but also on particle characteristics (e.g., size, shape, color). Thus, data processing has to evaluate large sets 
of spectral as well as particle-related data. Due to the large number of particles, we suggest using computational, highly automated solutions for time-efficient analysis. Instrument manufacturers are only beginning to offer particle analyzer programs that not only allow for the acquisition of large numbers of spectra automatically but also provide highperformance data-processing tools. Meanwhile, research groups have developed their own data-processing routines based on commercial software [104]. Furthermore, opensource solutions for both $\mu$-FTIR imaging (e.g., siMPle [79]) and RM (TUM-ParticleTyper [62]; see "Raman microspectroscopy") or for RM and $\mu$-FTIR spectroscopy (GEPARD [49]; see "Raman microspectroscopy") are used. In the following, general guidelines for spectra evaluation and reporting for any data-processing approach are described.

Regardless of the applied measurement method, large numbers of spectra are generated. They must be evaluated while assuring both correct identification and time-efficient processing. Ideally, the software tools do not only ensure equal treatment of all spectra of a dataset, but they also are fast and stable when processing large datasets and provide convenient formats of data output as well as (easy to use) tools for the operator to evaluate and double-check the identification of the automatically acquired spectra.

There are various approaches to the evaluation of spectra, such as correlation to spectral databases [29, 74, 105], modelbased classification [104], or descriptive methods (e.g., multivariate curve resolution-alternating least squares (MCRALS) and independent component analysis (ICA)) (El Rakwe et al., in prep.). Database searches rely on similarity measures, such as the absolute distance, the Euclidean distance, least squares [106], or the Pearson correlation coefficient to find an unknown spectrum's match in a database. For large datasets, the correlation of spectra to databases can become very slow or require high computational power. Reducing the number of spectra in the database can reduce the processing load. Nevertheless, database searches can reach method-intrinsic performance limits, especially for spectra from environmental samples with low signal-to-noise ratios or other deviations from the ideal polymer spectra. Alternatively, training a set of chemometric classifiers, i.e., of random decision forest (RDF) classifiers, in combination with applying spectral descriptors for data preprocessing was proposed $[64,104]$. Classifiers are complex algorithms that sort data into categories of information (classes). They learn to predict the class affiliations of unknown data from labeled training data. For the identification of polymer spectra, a training dataset is used which is comprised of a sufficient number of representatives from all the polymer classes that shall be detected. For further details, reference may be made to Hufnagl et al. [104]. Spectral classifiers or descriptive methods can reduce the computational demands, but their implementation is not trivial and becomes more challenging as the number of substance classes increases.

To date, the most common approach is the correlation of spectra to databases. During this process, the search parameters (e.g., raw or derivative spectra, spectral range, model of comparison [106]), data treatment (e.g., background correction, spectral normalization), and spectral quality can significantly influence the results [107]. Likely, the best control of whether a polymer spectrum has been identified correctly is an experienced spectroscopist's evaluation. Such a manual QC can, however, be time-consuming due to the large number of spectra. As an alternative, quality criteria for the automated spectral identification can be defined. Database software frequently provides a quantitative criterion for the similarity of a spectrum and a database spectrum, which is often referred to as the hit quality index (HQI). Using a HQI of $70 \%$ as a threshold for accepting a result as correct has been suggested $[68,108]$. Beside this absolute value, the HQI difference $(\Delta \mathrm{HQI})$ of the first to the second-best hit can be consulted, reflecting especially the selectivity of the correlation as pointed out by Renner et al. [109]. For this purpose, two suitable approaches to define a threshold exist: (i) $\Delta \mathrm{HQI} \geq 10 \%$ or (ii) $\Delta \mathrm{HQI} \geq \mathrm{HQI} \times 0.1$. However, both concepts will not allow distinguishing between similar polymers or subclasses of polymers like low and high density polyethylene (LDPE and HDPE) or polyamide 6 and polyamide 6.6 (PA 6 and PA 6.6) making re-evaluation by manual inspection necessary [109-111].

Furthermore, it has to be underlined that HQI values generated with different software might not be comparable, as the suppliers have implemented varying algorithms. Furthermore, the absolute HQI value depends on the quality of spectra and the type of sample (reference material or environmental sample). Consequently, the data pretreatment, the applied correlation algorithm, as well as the database to which the spectra were correlated needs to be reported in studies [109]. As the HQI depends on many different factors, a general numerical value cannot be defined as a quality criterion. If a laboratory chooses to use only the HQI as quality criterion, determination and validation of the HQI thresholds with respect to the instrumental capabilities and the analyzed sample types is obligatory.

To the best of our knowledge, there are no standard operation protocols on how to accomplish validation of HQI thresholds, but a thought experiment based on statistical hypothesis tests could be considered and is described in the ESM, section S3. It should be noted that it has not been tested in routine analysis, so its purpose is to provide the reader with a suggestion on how to approach the task. We strongly encourage the laboratories to thoroughly test and modify the approach and to share their findings.

Current experience is not yet sufficient to provide an assurance to readers that statistical hypothesis tests allow a failure- 
safe determination of a correct HQI but we encourage verifying one's HQI thresholds with a suitable approach instead of picking an unsubstantiated number.

If approaches other than correlating spectra to databases are used, these recognition models have to be validated and reported just as the HQI values described above.

As a standard set for spectral identification, we suggest to include the following main polymers: polyethylene (PE), poly (ethylene terephthalate) (PET), PP, PS, PC, poly (vinyl chloride) (PVC), poly (methyl methacrylate) (PMMA), PTFE, polyamide (PA, nylon), and polyurethanes (PU). The latter can possess a variety of side groups depending on the monomers they are made from, resulting in differing IR and Raman spectra. It is thus recommended that different kinds of PU are included into the set of standard polymers. Since spectra of polymers can differ significantly due to weathering of MPs, it is also advisable to include spectra of aged polymers in the database [112]. Each laboratory is furthermore recommended to augment this set with further polymers (e.g., acrylonitrile butadiene styrene (ABS), natural rubber, copolymers) as well as non-synthetic and/or non-polymeric materials that can commonly occur in samples (e.g., amorphous carbon, cellulose, proteins, inorganic materials such as quartz, and other minerals). As stated in "Raman microspectroscopy," Raman spectroscopy can identify additives such as pigments and dyes. In the case of these colorants, their typically sharp peaks can be of higher intensity than polymer peaks. Therefore, optimized measurement parameters have to be applied for identification of colorants/pigments and polymers. In order to recognize these potentially polymeric particles, the inclusion of a library of common dyes for polymers can be useful. Spectral libraries are commercially available, but many software tools for database search allow the creation of personalized libraries. This can be advantageous as database and sample spectra are obtained with the same instrument, and spectra of potential contaminants occurring just in a certain lab can be included [80]. Very recently, Cowger et al. developed a new open-source library (Open Specy) for MP identification for both measurement techniques, RM and IR. Regarding particle identification, it is fully comparable to commercial software. Users can upload and share their own spectra with the community, so that the library is continuously growing and improving [113].

The operator must pay attention to distinguish the spectra of PA and proteins. For both techniques, they can be distinguished well when comparing high-quality spectra. However, as spectra from environmental samples are often distorted, identification has to rely on the most prominent peaks, which are similar both in Raman and in (FT)IR spectroscopy. Detailed information on this difficulty can be found in the ESM, section S1. Molecules containing long carbon chains can have very similar IR and/or Raman spectra. This is the case, for example, between PE and stearates (e.g., found in latex gloves and used as food additives), stearamides (e.g., used as polymer additives and slipping agents) or sodium dodecyl sulfate (e.g., used as detergent) ( [45] and unpublished data, N. Benismail).

Overall, each laboratory is encouraged to augment these data-processing recommendations according to their needs. Details of the determination of the particle properties and the spectral identification must be reported as stated in "Ways of reporting results and valuable information."

\section{Method validation and quality controls}

In analytical chemistry, method performance is assured by the determination of QA and QC parameters. Both together prove that a given method is effective and reliable. These include the calculation of recovery rates, repeatability, reproducibility, limits of detection and quantification (LOD and LOQ), and blank contribution as well as overall robustness. In addition, ILC studies are necessary to check accuracy and precision of the methods used by different laboratories (see also Figure 1).

Method validation is an analytical challenge, especially in the case of MP analysis, and the following difficulties must be taken into account: Unlike other conventional contaminants, MPs are not dissolved in aqueous samples, but are present in particulate form. Therefore, they are not homogenously distributed and up to now, suitable certified reference materials are not available. As the expected concentration and size of MPs in clean water are very low, a mass-based approach cannot be regarded as promising (see "Introduction"). A first international ILC study on MPs in water has been initiated by the Joint Research Centre (JRC) of the European Union [21], but at the time of writing, the results and conclusions are still pending. They can be a basis for further international proficiency tests (PT).

In most studies on MPs, QA/QC data include solely the analysis of blanks for control of sample contamination. Wang et al. used pre-filtered drinking water as blank sample. After filtration through aluminum oxide filters (pore size 0.22 $\mu \mathrm{m}$ ) and analysis with SEM and RM, the blank contribution was $<5 \%$ of MP abundance [114]. Pivokonsky et al. used water of HPLC-grade and analyzed particles $>10 \mu \mathrm{m}$ with FTIR spectroscopy and particles $<10 \mu \mathrm{m}$ by RM. They also report a blank contribution $<5 \%$ [34]. On the other hand, Zhang et al. indicated that there was no blank contribution when filtering 11 ultrapure water through nitrocellulose filters (pore size $0.45 \mu \mathrm{m}$ ) with subsequent ATR- $\mu$-FTIR analysis [115]. Contrarily, filtration of 1501 of pre-filtered (with 3- $\mu \mathrm{m}$ stainless steel cartridge filters) drinking water using aluminum oxide filters (pore size $0.2 \mu \mathrm{m}$ ) resulted in a blank contribution of $45 \pm 22$ fibers of different colors [59]. Schymanski et al. reported a blank contribution of $14 \pm 13$ MPs (size down to 5 $\mu \mathrm{m}$ ) in 11 ultrapure water (system fed with deionized water, 
end filter with $0.22 \mu \mathrm{m}$ pore size) [33]. Predominant polymers detected were PET, PE, PS, and PP. When analyzing particles down to a size of $1 \mu \mathrm{m}$, even blank values of $384 \pm 468 \mathrm{MPs} / 1$ are common [32]. Furthermore, several authors report the procedures to prevent sample contamination during MP analysis [34, 52, 116, 117].

However, published papers seldom provide QA/QC data regarding efficiency, repeatability, and reproducibility or on LOD/ LOQ. As methods are not sufficiently validated, quantitative analysis is affected by the analytical technique used and its capacity to detect and identify MPs with confidence. So far, there are still important analytical limitations regarding the filtration step (choice of filters and sample volume) and the detection and the identification potential of analytical methods (IR spectroscopy/RM). In addition, there is still a certain subjectivity for data evaluation.

Overall, there is an urgent need to perform analysis under QA/QC management. This will lead to more uniform and comparable data. QA/QC measures of sampling, sample preparation, and detection will permit the development of a more realistic picture of MP occurrence, especially for small-sized MPs [20, 118].

One of the main steps of the QA/QC system is to characterize the methodology and to perform the initial validation of the method. For this specific methodology, we propose to assess the following important steps:

I. Verification of the size measurement capability of the equipment (minimum requirement)

II. Verification of qualitative polymer identification at the claimed minimal size (minimum requirement)

III. Determination of the LOD (minimum requirement)

IV. Verification of the recovery rates of the entire method (best practice)

V. Participation at interlaboratory comparisons and proficiency tests

\section{Verification of size measurement}

To prove that particles are being assigned correct size values, the size calibration of the particle detection should be performed with, for example, certified size standards or engraved plates/filters. Uncertified material can also be suitable, if the size is checked by optical microscopy. The particles with a certain size should be determined correctly within a tolerance of $\pm 10 \%$ [119], or, in case of imaging techniques which are tied to a certain pixel resolution, within a tolerance of \pm 1 pixel [64].

II. Verification of qualitative polymer identification at minimal size
Verification of qualitative polymer identification should be performed using the most commonly applied polymers (PE, PET, PC, PP, PVC, PS, etc.) and analyzing at the claimed minimal detectable size. For validation, MPs from real samples (with previously identified polymer type), commercially available polymer standards [61], or in-house-produced reference polymer particles $[64,69]$ may be used. Very recently, the JRC published their method on how they produced reference material for the above-mentioned ILC [120]. The lab is required to achieve goodquality spectra. If an automated database algorithm for polymer identification is used, acceptable quality indices for the library match should be documented (see HQI, "Data processing"). It is highly recommended to check whether the expected polymers, e.g., the plastic container/cap of bottled water can be recognized by the analysis. Otherwise, these spectra have to be added to the database or the analysis parameters must be adapted.

\section{Determination of the limit of detection}

The LOD is a global value demonstrating the capability of the laboratory to master its scientific equipment, filtration systems, but also the potential contamination of samples with MPs coming from the lab environment, air, operator, or lab materials [20].

The most challenging QA/QC step is the verification of the quantitative MP count. Usually, the LOD and LOQ are common parameters in analytical chemistry to describe the sensitivity of a method. The LOD describes the minimum amount of an analyte that can be detected but not necessarily quantified [121]. The smallest accurately quantifiable amount of an analyte is described by the LOQ. There are different methods to determine or calculate the LOD and LOQ for calibratable techniques of dissolved substances, but these are not applicable to solid particles like MPs, which cannot be diluted. In this case, the LOD of the method, which describes the smallest amount of detectable MPs in the sample, can be determined as the mean of blank samples plus the threefold standard deviation (minimum requirement; see Eq. 4). The determination of LOD based on blank values is in agreement with several recently published papers on microplastic analysis by $\mu$-FTIR $[53,64,122,123]$. We suggest using 10 blank samples to initially calculate the LOD. Ideally, this should be done for every particle size category/range that is given in the report with each LOD being expressed in relation to the size range values (e.g., LOD [5 $\mu \mathrm{m}]=8 \mathrm{MPs}$ ). In practice, quoting the LOD for the smallest size class is sufficient.

$L O D=$ mean $_{10 \text { blanks }}+3 * s_{10 \text { blanks }}$

Verification has to include the entire analytical process and has to take into account different polymers present in (blank) samples. Thus, LOD may be expressed as the undifferentiated sum of all polymers. For detailed scientific studies, polymerspecific LODs can be more advisable. 
Additionally, when the experience in MP analytics has grown, the LOQ should be calculated and reported as the mean of 10 blanks adding the tenfold standard deviation (best practice). This will provide accurate qualitative data for the contamination of water samples.

\section{Verification of the recovery rate of the entire method}

As long as there is no certified reference material and the possibility to prove the quality of the method with an ILC, the accuracy of a method is not fully provable [124]. Therefore, the recovery rates should be calculated based on the analysis of spiked samples (even with different levels of MPs) in the best practice approach [20]. Enders et al. determined the recovery rate of their entire extraction procedure with a new modified separation funnel for particle-rich samples with 60-80 fluorescent PE particles in the size range between 125 and $150 \mu \mathrm{m}$ [28]. They reported a recovery rate of $78 \pm 6 \%$. Although they published a protocol to extract MPs between $10 \mu \mathrm{m}$ and $5 \mathrm{~mm}$ from environmental samples, they did not determine the recovery rate for particles between 10-125 $\mu \mathrm{m}$ and $150 \mu \mathrm{m}-5 \mathrm{~mm}$. For a best practice routine, recovery rates should be determined for the whole particle size range targeted in the analysis. However, exact spiking of particle numbers with sizes $<100 \mu \mathrm{m}$ is still a challenge.

Analysis of standards for cytometers can be an alternative, if it is proven, that the system recognizes the spherical shapes correctly [119]. Another possibility is to use commercially available MPs of different sizes [61] or to produce own reference materials [69]. Applying the latter method, dispersed secondary MP reference particles can be generated via ultrasonic treatment. Nevertheless, there is still a high need for reference materials of different polymer types with a defined number, size or size range, and shape (incl. spheres, irregular fragments, films, and fibers). These would enable comprehensive validation and ILC studies for different methods.

To simplify, one can determine the recovery rate of the whole method with just one kind of polymer, assuming a similar behavior of main polymers during preparation and filtration steps of the method.

Once the important characteristics of the method are verified, the lab should complete the quality management of the method by systematically preparing blank (process) controls in each series of samples. These blank samples are necessary to demonstrate the correct sample handling and to ensure the validity of the results (see "Laboratory blanks").

V. Participation at interlaboratory comparisons and proficiency tests

An important step in the generalized acceptance of analytical methods is their testing through ILC. Currently, there are no widely accepted standardized methods and very few reports of ILC or PT undertaken to evaluate potential analytical MP procedures. In literature, a number of projects or consortia have reported the organization of restricted, internal ILC $[23,27,125]$. However, with the exception of the work of Isobe [126], where MP particles ranging from 0.4 to $5.7 \mathrm{~mm}$ were analyzed, the detailed outcome of the results is not openly available. With regard to ILC or PT studies, where participation is freely accessible to a wide range of laboratories, there are no fully documented studies at this time. But, recent initiatives by QUASIMEME [22] and subsequently JRC [21] provide examples of ongoing trials. These two examples neither propose predefined methods or protocols, but rather supply participating laboratories with one or more types of (as far as possible homogeneous and stable) MP-containing samples. These samples are analyzed by methods and procedures, which the participants judge appropriate to provide the requested data, e.g., plastic identity, particle number, and particle size. In both cases, the results and experience gained during the trials are intended to aid harmonization of methodologies while, in the latter case, an additional aim is to undertake the first steps toward the development and evaluation of potential MP reference standards.

While the lack of information from ILC studies is limiting method development and harmonization, there is a growing public and political awareness of MPs, which is driving legislation. It will require standardized methods, e.g., the recast of the EU Drinking Water Directive [127]. These legal drivers will increase the need for methods to be subjected to more rigorous QC tools, such as ILC and PT exercises. Quality control will in turn require general access to fit-for-purpose reference materials. The development of such materials will be a technically complex and time-consuming process, which presents many challenging issues. Firstly, defining how a reference material should be designed is a key issue given the huge range of polymer types, additives, shapes, sizes, and concentrations, which can be present in the many types of background matrices. For some applications, such as MPs in drinking water, the composition (regarding the variety of polymer types and size range and shape of MPs) may be simpler. For other sample types, such as sediments or municipal wastewater, test samples will be much more complex to define representatively. Secondly, it is likely that widely available standards would incorporate artificially manufactured MPs (e.g., mechanically milled or custom synthesized). These may present behavioral variations (agglomeration state, adhesion to filters and laboratory equipment) resulting from their surface properties (hydrophobicity, charge), which are not typical for MPs from real (environmental) samples. These differences in the behavior of MP fragments may influence overall sample recovery and, in the case of agglomeration, compromise particle counting results. One possibility to overcome these limitations is to generate dispersed secondary MP reference particles via ultrasound treatment [69]. Finally, 
verifying adequate homogeneity across sample batches and testing temporal stability will need reliable and reproducible procedures prior to performing more extended ILC trials. These will be necessary to assign consensus values (particle number, mass, size distribution, etc.) to the standards.

\section{Ways of reporting results and valuable information}

A report of MP analysis must contain all parameters of the sample, sample treatment (description of filtration, filter type, and pore sizes), and the analytical methods [17]. For the calculation of the total number of microplastics in the sample, subtracting the laboratory blank value of the sample series or the limit of reporting from the sample results is not recommended. The laboratory should indicate the value of LOD with the results. For (FT)IR spectroscopy, these are measurement mode, use of FPA detectors, spectral range, spectral resolution, and number of co-added spectra. Parameters for RM that need to be reported are as follows: optical imaging mode (bright or dark field), spatial resolution of the image (pixel size), microscopic objective (magnification and numerical aperture) for Raman measurement, confocality, wavelength of excitation laser and laser power at the sample, grating, focal length of the spectrometer, exposure time, and the number of co-added spectra. For both spectroscopic methods, the description and validation of the spectral identification routine have to be included. For communication of the analytical results, a report on the MP content of a sample obtained with spectroscopic methods must contain the following results:

- Total number of particles in the sample or sub-sample (see "Models for sub-sampling of particles deposited on a filter," if possible to obtain this value with the instrument used)

- In case of sub-sampling additionally: analyzed area of the filter $(\%)$ or statistical percentage of analyzed particles on the total number of particles (\%)

- Number of particles analyzed (obtained by measurement)

- LOD: preferably size-dependent (see "Method validation and quality controls" for more details)

- Number of total MPs identified (obtained by extrapolation or measurement)

- By type of polymer

- By size ranges

At present, it is common practice to report MP numbers in predefined size classes, i.e., in a binned form [10, 54, 81, 128, 129]. By this, the details on the size of each MP detected in samples get lost. However, these details are relevant for risk assessment. As the non-alignment of methods and reporting hinders risk evaluation [130], future studies should at least use the same size classes for binning, as proposed in Table 1 in the section on analysis: $1-5 \mu \mathrm{m}, 5-10 \mu \mathrm{m}, 10-20 \mu \mathrm{m}, 20-50 \mu \mathrm{m}$, 50-100 $\mu \mathrm{m}, 100-500 \mu \mathrm{m}$, and $>500 \mu \mathrm{m}$. Nevertheless, the future best practice should include the reporting of details on the particle size of all detected MPs, e.g., in the form of supplementary data or at least on demand.

As additional information or upon request, the report might contain:

- Details about blank samples (number, type of polymer, size range)

- Shape (e.g., fragment, fiber, sphere) and color of particles

- (Microscopic) images of measured filters

- Number of (potential) MPs not included in the minimum set of polymer classes proposed in "Data processing" (e.g., colored polymers, pigment or dye particles) sorted by type or by size range, shape, color, etc.

- Total number of non-plastic particles identified (e.g., minerals, proteins, cellulose) sorted by type or size range, shape, color, etc.

- Total number of non-identified particles

- Software and routines used for

- Determining particle properties

- Spectra processing

- Spectral identification; in the case of database search information about the database (commercially, homemade); if a HQI was used, the algorithm used and how the threshold was determined

Laboratories must only express quantitative results, if they exceed the LOD at the size limit of the method. Particles that are, e.g., smaller than the filter pores, can be found on a filter nevertheless, if deposited between the pores. The proportion of such particles retained is unknown, and thus, their number is not representative for the sample. Furthermore, blanks and possible cross-contaminations have not been evaluated for particles smaller than the size limit. However, these numbers can be of interest as an indicator of the amount of smaller particles in the sample. Thus, if a laboratory provides these quantitative values, it must demonstrate unambiguously that any of the particles counted are intrinsic to the sample in order to eliminate any risk of false-positive results, and state that this number is not representative for the sample.

\section{Discussion and conclusions}

The lack of harmonized methods and analytical standard substances and the difficulty to validate methods for the 
determination of MPs in clean water have led to highly variable and even sometimes contradictory data [15]. The proposed quality criteria by Koelmans and colleagues include the sampling method, sample size, sample processing and storage, laboratory preparation, clean air conditions, positive and negative controls, sample treatment, and polymer identification. The present consensus paper discusses and sums up details regarding the most important spectroscopic methods that can be used for MP analysis in clean water. All of the above-mentioned quality criteria were integrated in this guideline (see Table 1). It allows the reader to compare and evaluate existing studies. Furthermore, the guidelines can be used to better understand and thus make a more advantageous choice when setting up MP research studies. Given best practice approaches will contribute to a better harmonization of analytical methods for MP analysis in clean water samples down to $1 \mu \mathrm{m}$. A schematic overview of important precautions for MP analysis and sampling advices are given in Figure 1.

All these elements are intended to support the standardization processes throughout the different normalization committees. While this consensus paper from twelve European analytical laboratories and institutions has concentrated on (FT)IR/RM methods, for the purpose of monitoring as well as gaining a more comprehensive knowledge on MP contamination in food, water, air, and environmental samples, both spectroscopic and thermo-analytical methods are required. Therefore, it will also be important that a similar consideration be given to harmonizing thermo-analytical methods for MP detection. Above all, an ongoing exchange of scientists and laboratories, ILC studies with certified polymer standards and coordinating structures are required. These will pave the way to enable progress in the harmonization and standardization of MP detection and to allow for representative and reliable MP analysis in different environmental and food samples.

Supplementary Information The online version contains supplementary material available at https://doi.org/10.1007/s00216-021-03498-y.

Acknowledgements and funding B. E. Oßmann thanks the Bavarian State Ministry of the Environment and Consumer Protection for funding the projects "Detection of microplastics in selected foods" and "Expansion of the analytics of microplastics in food." S. Lacorte acknowledges funding from the Ministry of Science and Innovation of Spain (project PID2019-105732GBC21). J. Weisser, K. Glas, T. Hofmann, N. P. Ivleva, and E. von der Esch receive funding from the Bavarian Research Foundation (Bayerische Forschungsstiftung (BFS), AZ-1258-16 ("MiPAq"). N. P. Ivleva thanks the Federal Ministry of Education and Research (Bundesministerium für Bildung und Forschung (BMBF), projects

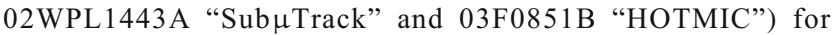

financial support. D. Fischer and F. Fischer acknowledge funding from the projects MicroCatch_Balt, PLASTRAT, PLAWES by the BMBF in the call "Plastics in the environment," as well as from the BONUS BLUE BALTIC project MICROPOLL, which has received funding from BONUS (Art 185), funded jointly by the EU and the BMBF. N. Zumbülte and C. S. Witzig acknowledge funding by the BMBF of the project "MicBin."

Funding Open Access funding enabled and organized by Projekt DEAL.

\section{Declarations}

Conflict of interest The authors declare no competing interests.

Open Access This article is licensed under a Creative Commons Attribution 4.0 International License, which permits use, sharing, adaptation, distribution and reproduction in any medium or format, as long as you give appropriate credit to the original author(s) and the source, provide a link to the Creative Commons licence, and indicate if changes were made. The images or other third party material in this article are included in the article's Creative Commons licence, unless indicated otherwise in a credit line to the material. If material is not included in the article's Creative Commons licence and your intended use is not permitted by statutory regulation or exceeds the permitted use, you will need to obtain permission directly from the copyright holder. To view a copy of this licence, visit http://creativecommons.org/licenses/by/4.0/.

\section{References}

1. Andrady AL. Microplastics in the marine environment. Mar. Pollut. Bull. 2011. https://doi.org/10.1016/j.marpolbul.2011.05. 030 .

2. Thompson RC, Olsen Y, Mitchell RP, Davis A, Rowland SJ, John AWG, et al. Lost at sea: where is all the plastic? Science. 2004. https://doi.org/10.1126/science.1094559.

3. Braun U, Jekel M, Gerdts G, Ivleva NP, Reiber J. Microplastics analytics: sampling, preparation and detection methods. Discussion Paper within the scope of the research focus "Plastics in the Environment: Sources, Sinks, Solutions"; 2018.

4. Hartmann NB, Hüffer T, Thompson RC, Hassellöv M, Verschoor A, Daugaard AE, et al. Are we speaking the same language? Recommendations for a definition and categorization framework for plastic debris. Environ Sci Technol. 2019. https://doi.org/10. 1021/acs.est.8b05297.

5. International Organization for Standardization (ISO). ISO/TR 21960:2020: Plastics - environmental aspects - state of knowledge and methodologies (21960:2020); 2020. https://www.iso.org/ standard/72300.html. Accessed 17 Feb 2021.

6. State Water Resources Control Board. Adoption of definition of 'microplastics in drinking water': resolution no. 2020-0021. 2021. https://www.waterboards.ca.gov/board decisions/adopted orders/resolutions/2020/rs2020_0021.pdf.

7. Koelmans AA, Besseling E, Shim WJ. Nanoplastics in the aquatic environment. Critical review. In: Bergmann M, Gutow L, Klages M, editors. Marine anthropogenic litter: Springer International Publishing; 2015. pp. 325-340. 
8. Ter Halle A, Jeanneau L, Martignac M, Jardé E, Pedrono B, Brach L, et al. Nanoplastic in the North Atlantic subtropical gyre. Environ Sci Technol. 2017. https://doi.org/10.1021/acs.est. $7 \mathrm{~b} 03667$.

9. Schwaferts C, Niessner R, Elsner M, Ivleva NP. Methods for the analysis of submicrometer- and nanoplastic particles in the environment. Trends Analyt. Chem. 2019. https://doi.org/10.1016/j. trac.2018.12.014

10. Winkler A, Santo N, Ortenzi MA, Bolzoni E, Bacchetta R, Tremolada P. Does mechanical stress cause microplastic release from plastic water bottles? Water Res. 2019. https://doi.org/10. 1016/j.watres.2019.115082.

11. Ivleva NP. Chemical analysis of microplastics and nanoplastics: challenges, advanced methods and perspectives. Chem Rev; submitted. 2021.

12. WHO. Microplastics in drinking-water. Geneva: World Health Organization; 2019.

13. State California. Health and Safety Code - California Safe Drinking Water Act [116270 - 116755].

14. European Parliament and the Council. Directive (EU) 2020/2184 of the European Parliament and of the Council of 16 December 2020 on the quality of water intended for human consumption (recast): OJ L 435, 23.12.2020, p. 1-62.

15. Koelmans AA, Mohamed Nor NH, Hermsen E, Kooi M, Mintenig SM, de France J. Microplastics in freshwaters and drinking water: critical review and assessment of data quality. Water Res. 2019. https://doi.org/10.1016/j.watres.2019.02.054.

16. Zarfl C. Promising techniques and open challenges for microplastic identification and quantification in environmental matrices. Anal Bioanal Chem. 2019. https://doi.org/10.1007/ s00216-019-01763-9.

17. Cowger W, Booth AM, Hamilton BM, Thaysen C, Primpke S, Munno K, et al. Reporting guidelines to increase the reproducibility and comparability of research on microplastics. Appl. Spectrosc. 2020. https://doi.org/10.1177/0003702820930292.

18. Hidalgo-Ruz V, Gutow L, Thompson RC, Thiel M. Microplastics in the marine environment: a review of the methods used for identification and quantification. Environ Sci Technol. 2012. https://doi.org/10.1021/es2031505.

19. Hermsen E, Mintenig SM, Besseling E, Koelmans AA. Quality criteria for the analysis of microplastic in biota samples: a critical review. Environ Sci Technol. 2018. https://doi.org/10.1021/acs. est.8b01611.

20. Brander SM, Renick VC, Foley MM, Steele C, Woo M, Lusher A, et al. Sampling and quality assurance and quality control: a guide for scientists investigating the occurrence of microplastics across matrices. Appl. Spectrosc. 2020. https://doi.org/10.1177/ 0003702820945713.

21. JRC. Call for laboratories to participate in proficiency tests on microplastics in drinking water and sediments. 2019. https://ec. europa.eu/jrc/en/science-update/call-laboratories-participateproficiency-tests-microplastics-drinking-water-and-sediments.

22. QUASIMEME. QUASIMEME study 2019. 2019. http://www. quasimeme.org/gfx_content/documents/QUASIMEME\% 20Interlaboratory\%20StudyPlastics\%20flyer.pdf.

23. Müller YK, Wernicke T, Pittroff M, Witzig CS, Storck FR, Klinger J, et al. Microplastic analysis - are we measuring the same? Results on the first global comparative study for microplastic analysis in a water sample. Anal Bioanal Chem. 2020. https://doi.org/10.1007/s00216-019-02311-1.

24. Jekel M, Anger P, Bannick CG, Barthel A-K, Braun U, Braunbeck T, Dittmar S, Eisentraut P, Elsner M, Gnirß R, Grummt T, Hanslik L, Huppertsberg S, Ivleva NP, Klöckner P, Knepper TP, Köhler H-R, Krais S, Kuckelkorn J, May E, Müller YK, Nießner R, Obermaier N, Oehlmann J, Pittroff M, Reemtsma T, Schmieg H, Schmitt T, Schür C, Storck FR, Strobel C, Triebskorn R, Wagner
M, Wagner S, Witzig CS, Zumbülte N, Ruhl AS. Mikroplastik im Wasserkreislauf: Universitätsverlag der TU Berlin; 2020.

25. Altmann K, Braun U, Fischer D, Fischer F, Ivleva NP, Sturm H, Witzig CS, Zumbülte N. QST1: Vergleichsversuch; 2021.

26. Braun U, Altmann K, Bannick CG, Becker R, Bitter H, Bochow M, et al. Mikroplastik-Analytik: Probenahme, Probenaufbereitung und Detektionsverfahren. Stand: November 2020; 2020.

27. BASEMAN-Project. BASEMAN-Project. http://www.jpi-oceans. eu/baseman/main-page. Accessed 17 Feb 2021.

28. Enders K, Lenz R, Ivar do Sul JA, Tagg AS, Labrenz M. When every particle matters: a QuEChERS approach to extract microplastics from environmental samples. MethodsX. 2020. https://doi.org/10.1016/j.mex.2020.100784.

29. Renner G, Schmidt TC, Schram J. Analytical methodologies for monitoring micro(nano)plastics: which are fit for purpose? Curr. Opin. Environ. Sci. Health. 2018. https://doi.org/10.1016/j.coesh. 2017.11.001.

30. Primpke S, Christiansen SH, Cowger W, de Frond H, Deshpande A, Fischer M, et al. Appl. Spectrosc. 2020. https://doi.org/10. $1177 / 0003702820921465$.

31. Ivleva NP, Wiesheu AC, Niessner R. Microplastic in aquatic ecosystems. Angew Chem Int Ed Engl. 2017. https://doi.org/10.1002/ anie. 201606957.

32. Oßmann BE, Sarau G, Holtmannspötter H, Pischetsrieder M, Christiansen SH, Dicke W. Small-sized microplastics and pigmented particles in bottled mineral water. Water Res. 2018. https://doi.org/10.1016/j.watres.2018.05.027.

33. Schymanski D, Goldbeck C, Humpf H-U, Fürst P. Analysis of microplastics in water by micro-Raman spectroscopy: release of plastic particles from different packaging into mineral water. Water Res. 2018. https://doi.org/10.1016/j.watres.2017.11.011.

34. Pivokonsky M, Cermakova L, Novotna K, Peer P, Cajthaml T, Janda V. Occurrence of microplastics in raw and treated drinking water. Sci. Total Environ. 2018. https://doi.org/10.1016/j. scitotenv.2018.08.102.

35. Stock V, Böhmert L, Lisicki E, Block R, Cara-Carmona J, Pack $\mathrm{LK}$, et al. Uptake and effects of orally ingested polystyrene microplastic particles in vitro and in vivo. Arch Toxicol. 2019. https://doi.org/10.1007/s00204-019-02478-7.

36. European Pharmacopoeia. Particulate contamination: sub-visible particles; 1/ Ph. Eur. 2.9.19: reference 01/2005:20919. 5th Edition (official on January 2005).

37. Hermsen E, Pompe R, Besseling E, Koelmans AA. Detection of low numbers of microplastics in North Sea fish using strict quality assurance criteria. Mar. Pollut. Bull. 2017. https://doi.org/10. 1016/j.marpolbul.2017.06.051.

38. Wiesheu AC, Anger PM, Baumann T, Niessner R, Ivleva NP. Raman microspectroscopic analysis of fibers in beverages. Anal. Methods. 2016. https://doi.org/10.1039/C6AY01184E.

39. Prata JC, da Costa JP, Duarte AC, Rocha-Santos T. Methods for sampling and detection of microplastics in water and sediment: a critical review. Trends Analyt. Chem. 2019. https://doi.org/10. 1016/j.trac.2018.10.029.

40. Toussaint B, Raffael B, Angers-Loustau A, Gilliland D, Kestens $\mathrm{V}$, Petrillo M, et al. Review of micro- and nanoplastic contamination in the food chain. Food Addit. Contam. Part A Chem. Anal. Control Expo. Risk Assess. 2019. https://doi.org/10.1080/ 19440049.2019.1583381.

41. Wesch C, Bredimus K, Paulus M, Klein R. Towards the suitable monitoring of ingestion of microplastics by marine biota: a review. Environ Pollut. 2016. https://doi.org/10.1016/j.envpol.2016.08. 076.

42. Wesch C, Elert AM, Wörner M, Braun U, Klein R, Paulus M. Assuring quality in microplastic monitoring: about the value of clean-air devices as essentials for verified data. Sci Rep. 2017. https://doi.org/10.1038/s41598-017-05838-4. 
43. International Organization for Standardization (ISO). ISO $14644-$ 1:2015: Cleanrooms and associated controlled environments - Part 1: Classification of air cleanliness by particle concentration: ISO(14644-1); 2015. https://www.iest.org/Standards-RPs/ISOStandards/ISO-14644-Series/ISO-14644-1. Accessed 16 Mar 2021.

44. Lei K, Qiao F, Liu Q, Wei Z, Qi H, Cui S, et al. Microplastics releasing from personal care and cosmetic products in China. Mar. Pollut. Bull. 2017. https://doi.org/10.1016/j.marpolbul.2017.09. 016.

45. Witzig CS, Földi C, Wörle K, Habermehl P, Pittroff M, Müller YK, et al. When good intentions go bad-false positive microplastic detection caused by disposable gloves. Environ Sci Technol. 2020. https://doi.org/10.1021/acs.est.0c03742.

46. Li L, Zhao X, Li Z, Song K. COVID-19: Performance study of microplastic inhalation risk posed by wearing masks. J Hazard Mater. 2020. https://doi.org/10.1016/j.jhazmat.2020.124955.

47. Renzi M, Guerranti C, Blašković A. Microplastic contents from maricultured and natural mussels. Mar. Pollut. Bull. 2018. https:// doi.org/10.1016/j.marpolbul.2018.04.035.

48. Gündoğdu S. Contamination of table salts from Turkey with microplastics. Food Addit. Contam. Part A Chem. Anal. Control Expo. Risk Assess. 2018. https://doi.org/10.1080/19440049. 2018.1447694.

49. Brandt J, Bittrich L, Fischer F, Kanaki E, Tagg A, Lenz R, et al. High-Throughput analyses of microplastic samples using Fourier transform infrared and Raman spectrometry. Appl. Spectrosc. 2020. https://doi.org/10.1177/0003702820932926.

50. Dris R, Gasperi J, Rocher V, Tassin B. Synthetic and nonsynthetic anthropogenic fibers in a river under the impact of Paris Megacity: sampling methodological aspects and flux estimations. Sci. Total Environ. 2018. https://doi.org/10.1016/j. scitotenv.2017.11.009.

51. Lusher AL, Munno K, Hermabessiere L, Carr S. Isolation and extraction of microplastics from environmental samples: an evaluation of practical approaches and recommendations for further harmonization. Appl. Spectrosc. 2020. https://doi.org/10.1177/ 0003702820938993.

52. Weber F, Kerpen J, Wolff S, Langer R, Eschweiler V. Investigation of microplastics contamination in drinking water of a German city. Sci. Total Environ. 2021. https://doi.org/10. 1016/j.scitotenv.2020.143421

53. Johnson AC, Ball H, Cross R, Horton AA, Jürgens MD, Read DS, et al. Identification and quantification of microplastics in potable water and their sources within water treatment works in England and Wales. Environ Sci Technol. 2020. https://doi.org/10.1021/ acs.est.0c03211.

54. Kirstein IV, Hensel F, Gomiero A, Iordachescu L, Vianello A, Wittgren HB, et al. Drinking plastics? - Quantification and qualification of microplastics in drinking water distribution systems by $\mu$ FTIR and Py-GCMS. Water Res. 2021. https://doi.org/10.1016/ j.watres.2020.116519.

55. Schymanski D, Goldbeck C, Fürst P, Humpf H-U. Kunststoffpartikel sind überall - auch in Lebensmitteln? Nachr. Chem. 2016. https://doi.org/10.1002/nadc.20164052892.

56. Petersen L, Minkkinen P, Esbensen KH. Representative sampling for reliable data analysis: theory of sampling. Chemometr. Intell. Lab. Syst. 2005. https://doi.org/10.1016/j.chemolab.2004.09.013.

57. Kankanige D, Babel S. Smaller-sized micro-plastics (MPs) contamination in single-use PET-bottled water in Thailand. Sci. Total Environ. 2020. https://doi.org/10.1016/j.scitotenv.2020.137232.

58. Shruti VC, Pérez-Guevara F, Kutralam-Muniasamy G. Metro station free drinking water fountain- a potential "microplastics hotspot" for human consumption. Environ Pollut. 2020. https:// doi.org/10.1016/j.envpol.2020.114227.
59. Mintenig SM, Löder MGJ, Primpke S, Gerdts G. Low numbers of microplastics detected in drinking water from ground water sources. Sci. Total Environ. 2019. https://doi.org/10.1016/j. scitotenv.2018.08.178

60. Pittroff M, Müller YK, Witzig CS, Scheurer M, Storck FR, Zumbülte N. Microplastic analysis in drinking water based on fractionated filtration sampling and Raman microspectroscopy. Environ Sci Pollut Res Int. 2021. https://doi.org/10.1007/ s11356-021-12467-y.

61. Oßmann BE, Sarau G, Schmitt SW, Holtmannspötter H, Christiansen SH, Dicke W. Development of an optimal filter substrate for the identification of small microplastic particles in food by micro-Raman spectroscopy. Anal Bioanal Chem. 2017. https:// doi.org/10.1007/s00216-017-0358-y.

62. von der Esch E, Kohles AJ, Anger PM, Hoppe R, Niessner R, Elsner M, et al. TUM-ParticleTyper: a detection and quantification tool for automated analysis of (Microplastic) particles and fibers. PLoS ONE. 2020. https://doi.org/10.1371/journal.pone.0234766.

63. Primpke S, Dias AP, Gerdts G, et al. Anal. Methods. 2019. https:// doi.org/10.1039/C9AY00126C.

64. Weisser J, Beer I, Hufnagl B, Hofmann T, Lohninger H, Ivleva $\mathrm{NP}$, et al. From the well to the bottle: identifying sources of microplastics in mineral water. Water. 2021. https://doi.org/10. 3390/w13060841.

65. Löder M, Gerdts G. Methodology used for the detection and identification of microplastics - a critical appraisal. In: Bergmann M, Gutow L, Klages M, editors. Marine anthropogenic litter: Springer International Publishing; 2015. pp. 201-227.

66. Käppler A, Windrich F, Löder MGJ, Malanin M, Fischer D, Labrenz M, et al. Identification of microplastics by FTIR and Raman microscopy: a novel silicon filter substrate opens the important spectral range below $1300 \mathrm{~cm}(-1)$ for FTIR transmission measurements. Anal Bioanal Chem. 2015. https://doi.org/10. 1007/s00216-015-8850-8

67. van Cauwenberghe L, Janssen CR. Microplastics in bivalves cultured for human consumption. Environ. Pollut. 2014. https://doi. org/10.1016/j.envpol.2014.06.010.

68. Yang D, Shi H, Li L, Li J, Jabeen K, Kolandhasamy P. Microplastic pollution in table salts from china. Environ Sci Technol. 2015. https://doi.org/10.1021/acs.est.5b03163.

69. von der Esch E, Lanzinger M, Kohles AJ, Schwaferts C, Weisser J, Hofmann T, et al. Simple generation of suspensible secondary microplastic reference particles via ultrasound treatment. Front Chem. 2020. https://doi.org/10.3389/fchem.2020.00169.

70. Cabernard L, Roscher L, Lorenz C, Gerdts G, Primpke S. Comparison of Raman and Fourier transform infrared spectroscopy for the quantification of microplastics in the aquatic environment. Environ Sci Technol. 2018. https://doi.org/10.1021/acs.est. $8 b 03438$.

71. Simon M, van Alst N, Vollertsen J. Quantification of microplastic mass and removal rates at wastewater treatment plants applying focal plane array (FPA)-based Fourier transform infrared (FT-IR) imaging. Water Res. 2018. https://doi.org/10.1016/j.watres.2018.05.019.

72. Löder MGJ, Kuczera M, Mintenig S, Lorenz C, Gerdts G. Focal plane array detector-based micro-Fourier-transform infrared imaging for the analysis of microplastics in environmental samples. Environ. Chem. 2015. https://doi.org/10.1071/EN14205.

73. Vianello A, Jensen RL, Liu L, Vollertsen J. Simulating human exposure to indoor airborne microplastics using a breathing thermal manikin. Sci Rep. 2019. https://doi.org/10.1038/s41598-019-45054-w.

74. Primpke S, Wirth M, Lorenz C, Gerdts G. Reference database design for the automated analysis of microplastic samples based on Fourier transform infrared (FTIR) spectroscopy. Anal Bioanal Chem. 2018. https://doi.org/10.1007/s00216-018-1156-x.

75. Primpke S, Fischer M, Lorenz C, Gerdts G, Scholz-Böttcher BM. Comparison of pyrolysis gas chromatography/mass spectrometry 
and hyperspectral FTIR imaging spectroscopy for the analysis of microplastics. Anal Bioanal Chem. 2020. https://doi.org/10.1007/ s00216-020-02979-w.

76. Liu F, Olesen KB, Borregaard AR, Vollertsen J. Microplastics in urban and highway stormwater retention ponds. Sci. Total Environ. 2019. https://doi.org/10.1016/j.scitotenv.2019.03.416.

77. Renner G, Schmidt TC, Schram J. Characterization and quantification of microplastics by infrared spectroscopy. In: Rocha-Santos TAP, Duarte AC, editors. Characterization and analysis of microplastics, vol. 75. Amsterdam: Elsevier; 2017. p. 67-118.

78. Harrison JP, Ojeda JJ, Romero-González ME. The applicability of reflectance micro-Fourier-transform infrared spectroscopy for the detection of synthetic microplastics in marine sediments. Sci. Total Environ. 2012. https://doi.org/10.1016/j.scitotenv.2011.11.078.

79. Primpke S, Cross RK, Mintenig SM, Simon M, Vianello A, Gerdts G, et al. Toward the systematic identification of microplastics in the environment: Evaluation of a new independent software tool (siMPle) for spectroscopic analysis. Appl. Spectrosc. 2020. https://doi.org/10.1177/0003702820917760.

80. Cowger W, Gray A, Christiansen SH, de Frond H, Deshpande A, Hermabessiere L, et al. Critical review of processing and classification techniques for images and spectra in microplastic research. Appl. Spectrosc. 2020.https://doi.org/10.1177/ 0003702820929064.

81. Primpke S, Godejohann M, Gerdts G. Rapid identification and quantification of microplastics in the environment by quantum cascade laser-based hyperspectral infrared chemical imaging. Environ Sci Technol. 2020. https://doi.org/10.1021/acs.est. 0c05722.

82. Li Q, Zeng A, Jiang X, Gu X. Are microplastics correlated to phthalates in facility agriculture soil? J Hazard Mater. 2021. https://doi.org/10.1016/j.jhazmat.2021.125164.

83. Ng EL, Lin SY, Dungan AM, Colwell JM, Ede S, Huerta Lwanga E, et al. Microplastic pollution alters forest soil microbiome. J Hazard Mater. 2021. https://doi.org/10.1016/j.jhazmat.2020. 124606.

84. Mughini-Gras L, van der Plaats RQJ, van der Wielen PWJJ, Bauerlein PS, de Roda Husman AM. Water Res. 2021. https:// doi.org/10.1016/j.watres.2021.116852.

85. Scircle A, Cizdziel JV, Tisinger L, Anumol T, Robey D. Occurrence of microplastic pollution at oyster reefs and other coastal sites in the Mississippi Sound, USA: impacts of freshwater inflows from flooding. Toxics. 2020. https://doi.org/10.3390/ toxics8020035.

86. Käppler A, Fischer D, Oberbeckmann S, Schernewski G, Labrenz $\mathrm{M}$, Eichhorn K-J, et al. Analysis of environmental microplastics by vibrational microspectroscopy: FTIR, Raman or both? Anal Bioanal Chem. 2016. https://doi.org/10.1007/s00216-016-9956-3.

87. Anger PM, von der Esch E, Baumann T, Elsner M, Niessner R, Ivleva NP. Raman microspectroscopy as a tool for microplastic particle analysis. Trends Analyt. Chem. 2018. https://doi.org/10. 1016/j.trac.2018.10.010.

88. Frère L, Paul-Pont I, Moreau J, Soudant P, Lambert C, Huvet A, et al. A semi-automated Raman micro-spectroscopy method for morphological and chemical characterizations of microplastic litter. Mar. Pollut. Bull. 2016. https://doi.org/10.1016/j.marpolbul. 2016.10.051.

89. Domogalla-Urbansky J, Anger PM, Ferling H, Rager F, Wiesheu AC, Niessner R, et al. Raman microspectroscopic identification of microplastic particles in freshwater bivalves (Unio pictorum) exposed to sewage treatment plant effluents under different exposure scenarios. Environ Sci Pollut Res Int. 2019. https://doi.org/10. 1007/s11356-018-3609-3.

90. Jakubek RS, Fries MD. Calibration of Raman wavenumber in large Raman images using a mercury-argon lamp. J. Raman Spectrosc. 2020. https://doi.org/10.1002/jrs.5887.
91. Gy P. Sampling of particulate materials theory and practice. Developments in geomathematics, vol. 4. Burlington: Elsevier Science; 1979.

92. Thaysen C, Munno K, Hermabessiere L, Rochman CM. Towards Raman automation for microplastics: developing strategies for particle adhesion and filter subsampling. Appl. Spectrosc. 2020. https://doi.org/10.1177/0003702820922900.

93. Minkkinen PO, Esbensen KH. Sampling of particulate materials with significant spatial heterogeneity - theoretical modification of grouping and segregation factors involved with correct sampling errors: fundamental sampling error and grouping and segregation error. Anal Chim Acta. 2019. https://doi.org/10.1016/j.aca.2018. 10.056 .

94. Brandt J, Fischer F, Kanaki E, Enders K, Labrenz M, Fischer D. Assessment of subsampling strategies in microspectroscopy of environmental microplastic samples. Front. Environ. Sci. 2021. https://doi.org/10.3389/fenvs.2020.579676.

95. Schwaferts C, Schwaferts P, von der Esch E, Elsner M, Ivleva NP. Which particles to select, and if yes, how many?: subsampling methods for Raman microspectroscopic analysis of very small microplastic. Anal Bioanal Chem. 2021. https://doi.org/10.1007/ s00216-021-03326-3.

96. Sobhani Z, Zhang X, Gibson C, Naidu R, Megharaj M, Fang C. Identification and visualisation of microplastics/nanoplastics by Raman imaging (i): down to $100 \mathrm{~nm}$. Water Res. 2020. https:// doi.org/10.1016/j.watres.2020.115658.

97. Fang C, Sobhani Z, Zhang X, Gibson CT, Tang Y, Naidu R. Identification and visualisation of microplastics/ nanoplastics by Raman imaging (ii): smaller than the diffraction limit of laser? Water Res. 2020. https://doi.org/10.1016/j.watres.2020.116046.

98. Gillibert R, Balakrishnan G, Deshoules Q, Tardivel M, Magazzù A, Donato MG, et al. Raman tweezers for small microplastics and nanoplastics identification in seawater. Environ Sci Technol. 2019. https://doi.org/10.1021/acs.est.9b03105.

99. Schwaferts C, Sogne V, Welz R, Meier F, Klein T, Niessner R, et al. Nanoplastic analysis by online coupling of Raman microscopy and field-flow fractionation enabled by optical tweezers. Anal. Chem. 2020. https://doi.org/10.1021/acs.analchem. 9b05336.

100. Dazzi A, Prater CB. AFM-IR: Technology and Applications in Nanoscale Infrared Spectroscopy and Chemical Imaging. Chem Rev. 2017. https://doi.org/10.1021/acs.chemrev.6b00448.

101. Meyns M, Primpke S, Gerdts G. Library based identification and characterisation of polymers with nano-FTIR and IR-sSNOM imaging. Anal. Methods. 2019. https://doi.org/10.1039/ C9AY01193E.

102. Zhou X-X, Liu R, Hao L-T, Liu J-F. Identification of polystyrene nanoplastics using surface enhanced Raman spectroscopy. Talanta. 2021. https://doi.org/10.1016/j.talanta.2020.121552.

103. Kurouski D, Dazzi A, Zenobi R, Centrone A. Infrared and Raman chemical imaging and spectroscopy at the nanoscale. Chem Soc Rev. 2020. https://doi.org/10.1039/C8CS00916C.

104. Hufnagl B, Steiner D, Renner E, Löder MGJ, Laforsch C, Lohninger $\mathrm{H}$. A methodology for the fast identification and monitoring of microplastics in environmental samples using random decision forest classifiers. Anal. Methods. 2019. https://doi.org/ 10.1039/c9ay00252a.

105. Primpke S, Lorenz C, Rascher-Friesenhausen R, Gerdts G. An automated approach for microplastics analysis using focal plane array (FPA) FTIR microscopy and image analysis. Anal. Methods. 2017. https://doi.org/10.1039/C6AY02476A.

106. Kwiatkowski A, Gnyba M, Smulko J, Wierzba P. Algorithms of chemicals detection using Raman spectra. Metrology and Measurement Systems. 2010. https://doi.org/10.2478/v10178010-0045-1. 
107. Liland KH, Almøy T, Mevik B-H. Optimal choice of baseline correction for multivariate calibration of spectra. Appl. Spectrosc. 2010;64:1007-16.

108. Woodall LC, Sanchez-Vidal A, Canals M, Paterson GLJ, Coppock R, Sleight V, et al. The deep sea is a major sink for microplastic debris. R. Soc. Open Sci. 2014. https://doi.org/10. 1098/rsos. 140317.

109. Renner G, Nellessen A, Schwiers A, Wenzel M, Schmidt TC, Schram J. Data preprocessing \& evaluation used in the microplastics identification process: a critical review \& practical guide. Trends Analyt. Chem. 2019. https://doi.org/10.1016/j.trac.2018.12.004.

110. Kroon F, Motti C, Talbot S, Sobral P, Puotinen M. A workflow for improving estimates of microplastic contamination in marine waters: a case study from North-Western Australia. Environ Pollut. 2018. https://doi.org/10.1016/j.envpol.2018.03.010.

111. Smith BC. Fundamentals of Fourier transform infrared spectroscopy: CRC Press; 2011.

112. Munno K, de Frond H, O'Donnell B, Rochman CM. Increasing the accessibility for characterizing microplastics: introducing new application-based and spectral libraries of plastic particles (SLoPP and SLoPP-E). Anal. Chem. 2020. https://doi.org/10.1021/acs. analchem.9b03626.

113. Cowger W, Steinmetz Z, Gray A, Munno K, Lynch J, Hapich H, et al. Microplastic spectral classification needs an open source community: Open Specy to the rescue! Anal. Chem. 2021. https://doi.org/10.1021/acs.analchem.1c00123.

114. Wang Z, Lin T, Chen W. Occurrence and removal of microplastics in an advanced drinking water treatment plant (ADWTP). Sci. Total Environ. 2020. https://doi.org/10.1016/j.scitotenv.2019. 134520

115. Zhang M, Li J, Ding H, Ding J, Jiang F, Ding NX, et al. Distribution characteristics and influencing factors of microplastics in urban tap water and water sources in Qingdao. China. Anal. Lett. 2020. https://doi.org/10.1080/00032719.2019. 1705476

116. Woodall LC, Gwinnett C, Packer M, Thompson RC, Robinson LF, Paterson GLJ. Using a forensic science approach to minimize environmental contamination and to identify microfibres in marine sediments. Mar. Pollut. Bull. 2015. https://doi.org/10.1016/j. marpolbul.2015.04.044.

117. Kosuth M, Mason SA, Wattenberg EV. Anthropogenic contamination of tap water, beer, and sea salt. PLoS ONE. 2018. https:// doi.org/10.1371/journal.pone.0194970.

118. Eerkes-Medrano D, Leslie HA, Quinn B. Microplastics in drinking water: a review and assessment. Curr. Opin. Environ. Sci. Health. 2019. https://doi.org/10.1016/j.coesh.2018.12.001.

119. Schymanski D, Humpf H-U, Fürst P. Determination of particle abrasion through milling with five different salt grinders by micro-Raman spectroscopy - a preliminary study with efforts towards improved quality control of the analytical methods. Food
Addit. Contam. Part A Chem. Anal. Control Expo. Risk Assess. 2020. https://doi.org/10.1080/19440049.2020.1748724.

120. Seghers J, Stefaniak EA, La Spina R, Cella C, Mehn D, Gilliland $\mathrm{D}$, et al. Preparation of a reference material for microplastics in water-evaluation of homogeneity. Anal Bioanal Chem. 2021. https://doi.org/10.1007/s00216-021-03198-7.

121. Cammann K. editor. Instrumentelle analytische Chemie: Verfahren, Anwendungen und Qualitätssicherung. Spektrum Lehrbuch. Heidelberg: Spektrum Akademischer Verlag; 2001.

122. Horton AA, Cross RK, Read DS, Jürgens MD, Ball HL, Svendsen $\mathrm{C}$, et al. Semi-automated analysis of microplastics in complex wastewater samples. Environ Pollut. 2020. https://doi.org/10. 1016/j.envpol.2020.115841.

123. Waddell EN, Lascelles N, Conkle JL. Microplastic contamination in Corpus Christi Bay blue crabs. Callinectes sapidus. Limnol Oceanogr. 2020. https://doi.org/10.1002/lol2.10142.

124. Filella M. Questions of size and numbers in environmental research on microplastics: methodological and conceptual aspects. Environ. Chem. 2015. https://doi.org/10.1071/EN15012.

125. SCCWRP-Project. SCCWRP-Project. https://www.sccwrp.org/ news/international-study-kicks-off-to-standardize-microplasticsmonitoring-methods/.

126. Isobe A, Buenaventura NT, Chastain S, Chavanich S, Cózar A, DeLorenzo M, et al. An interlaboratory comparison exercise for the determination of microplastics in standard sample bottles. Mar. Pollut. Bull. 2019. https://doi.org/10.1016/j.marpolbul.2019.07.033.

127. European Parliament and of the Council on the quality of water intended for human consumption. Proposal for a Directive of the European Parliament and of the Council on the quality of water intended for human consumption (recast) - Political agreement ST 60602020 REV 1, vol. 2020.

128. Tekman MB, Wekerle C, Lorenz C, Primpke S, Hasemann C, Gerdts G, et al. Tying up loose ends of microplastic pollution in the Arctic: distribution from the sea surface through the water column to deep-sea sediments at the HAUSGARTEN Observatory. Environ Sci Technol. 2020. https://doi.org/10. 1021/acs.est.9b06981.

129. Pivokonský M, Pivokonská L, Novotná K, Čermáková L, Klimtová M. Occurrence and fate of microplastics at two different drinking water treatment plants within a river catchment. Sci. Total Environ. 2020. https://doi.org/10.1016/j.scitotenv.2020. 140236.

130. Koelmans AA, Redondo-Hasselerharm PE, Mohamed Nor NH, Kooi M. Solving the nonalignment of methods and approaches used in microplastic research to consistently characterize risk. Environ Sci Technol. 2020. https://doi.org/10.1021/acs.est. 0c02982.

Publisher's note Springer Nature remains neutral with regard to jurisdictional claims in published maps and institutional affiliations. 